\title{
Localized meshless point collocation method for time-dependent magnetohydrodynamics flow through pipes under a variety of wall conductivity conditions
}

\author{
V. C. Loukopoulos - G. C. Bourantas - E. D. Skouras • \\ G. C. Nikiforidis
}

Received: 18 May 2010 / Accepted: 17 August 2010 / Published online: 8 September 2010

(C) Springer-Verlag 2010

\begin{abstract}
In this article a numerical solution of the time dependent, coupled system equations of magnetohydrodynamics (MHD) flow is obtained, using the strong-form local meshless point collocation (LMPC) method. The approximation of the field variables is obtained with the moving least squares (MLS) approximation. Regular and irregular nodal distributions are used. Thus, a numerical solver is developed for the unsteady coupled MHD problems, using the collocation formulation, for regular and irregular cross sections, as are the rectangular, triangular and circular. Arbitrary wall conductivity conditions are applied when a uniform magnetic field is imposed at characteristic directions relative to the flow one. Velocity and induced magnetic field across the section have been evaluated at various time intervals for several Hartmann numbers (up to $10^{5}$ ) and wall conductivities. The numerical results of the strong-form MPC method are compared with those obtained using two weak-form meshless methods, that is, the local boundary integral equation (LBIE) meshless method and the meshless local PetrovGalerkin (MLPG) method, and with the analytical solutions,
\end{abstract}

V. C. Loukopoulos $(\bowtie)$

Department of Physics, University of Patras, Rion,

26500 Patras, Greece

e-mail: vxloukop@physics.upatras.gr

G. C. Bourantas · G. C. Nikiforidis

Department of Medical Physics, School of Medicine,

University of Patras, Rion, 26500 Patras, Greece

E. D. Skouras

Department of Chemical Engineering, University of Patras,

Rion, 26500 Patras, Greece

\section{E. D. Skouras}

Institute of Chemical Engineering and High Temperature Chemical Processes, Foundation for Research and Technology,

Rion, P.O. Box 1414, 26504 Patras, Greece where they are available. Furthermore, the accuracy of the method is assessed in terms of the error norms $L_{2}$ and $L_{\infty}$, the number of nodes in the domain of influence and the time step length depicting the convergence rate of the method. Run time results are also presented demonstrating the efficiency and the applicability of the method for real world problems.

Keywords Local meshless point collocation .

Moving least squares · Unsteady MHD - Wall conductivity · LBIE · MLPG

\section{Introduction}

In the fields of physics and engineering, a challenging and popular problem, which often arising, is the flow of an incompressible, viscous, and electrically conducting fluid in a channel with partially conducting and partially non-conducting walls under a uniform transverse magnetic field. The aforementioned problem has many practical applications in the field of magnetohydrodynamics (MHD).

Magnetohydrodynamics studies the motion of electrically conducting fluids in the presence of magnetic fields. More precisely, from the physical point of view, the magnetic field influences the fluid motion, while, the fluid motion changes the magnetic field. Thus, the governing equations are inherently coupled in terms of the fluid velocity and the induced magnetic field. For the first time Hartmann [1] investigated the MHD flow of a viscous, incompressible, electrically conducting fluid between two parallel plates in the presence of a transverse magnetic field. Since then, a number of researches have investigated the flow of an electrically conducting fluid through channels (ducts) because of its important applications in MHD generators, pumps, accelerators, flow-meters, astrophysics, geology, power generation, thermonuclear 
reactor technology, designing cooling systems with liquid metals, medicine (biofluids and drug delivery), etc. It is not surprising that a lot of theoretical and experimental work has been carried out in this direction during the last decades.

For a limited number of MHD flow problems the solution can be obtained analytically $[2,3]$. These analytical solutions are usually available only for some non-complex geometries, subject to simple boundary conditions. However, the majority of the problems arising in the real world are difficult to solve in an analytical way, making the use of numerical methods inevitable. In the field of numerical computations several numerical techniques were developed and applied in order to solve this complex physical problem. Thus, numerous numerical methods such as FDM, FEM, closely related FVM and BEM were used for both steady and time-dependent MHD flows. More precisely, for steady state problems the authors in [4,5] have used FDM to solve MHD flow through channels of triangular cross-section for small values of the Hartmann number. Regarding FEM, solutions were presented in [6-8] for arbitrary cross-section ducts for Hartmann numbers less than $M=10$. Moreover, authors in [9] extended these studies to moderate Hartmann numbers up to $M=100$, using standard FEM with linear and quadratic elements. Furthermore, at [10] the analytical finite element method has been used to obtain a numerical solution in the limit of the Hartmann number $M \leq 1,000$. Additionally, FEM solution obtained for high values of $M$ using very fine mesh within the Hartmann layers [11]. Thus, it was possible to increase $M$ up to 1,000 and also to use general wall conductivities. Moreover, in [12] a fundamental solution was derived for coupled magnetohydrodynamic flow equations and results were presented for Hartmann number $M \leq 300$. Alternatively, BEM have been applied for solving MHD duct flow, however several problems have risen from the difficulties of solving huge systems and high computational costs due to the domain discretization. Papers at [13-17] are representative studies on the BEM solutions of MHD duct flow problems. All these BEM solutions have been obtained for small and moderate values of Hartmann number $(M \leq 50)$. Furthermore, authors in [18] using the finite element method obtained numerical results for very high values of Hartmann number up to $M=10^{5}$.

Numerical solutions of time-dependent MHD flow equations have been given in two [19] and three dimensions [20], using finite element methods. In [21] authors proposed a FDM scheme for three-dimensional unsteady MHD flows along with a temperature variation using an explicit RungeKutta method for step-by-step computations in time. Additionally, in [22] a convection-diffusion-reaction model was presented for solving unsteady MHD flow applying an FDM on non-staggered grids with a transport scheme in each ADI (predictor-corrector) spatial sweep. The solution algorithm in each of these unsteady MHD flow studies is based on explicit time-stepping schemes starting with the given initial conditions. Thus, the time increment must be taken very small to deal with the stability problems, therefore they are computationally expensive. A numerical scheme which is a combination of the dual reciprocity boundary element method (DRBEM) in space and the differential quadrature method (DQM) in time has been proposed in [23], for solving unsteady MHD flow problems in a rectangular duct with insulating walls. The solution procedure can be used with large time intervals to obtain the solution directly at the required time value. Computations have been carried out for moderate values of the Hartmann number.

Over the last decade, owing to the difficulty of the traditional numerical schemes in the mesh generation, new numerical methods, generally called "meshless" methods (also called "meshfree" methods), have been developed. Thus, meshless methods emerged as a potential alternative for solutions in computational mechanics, and a variety of such approaches have appeared. Several meshfree methods have been proposed; a review of the relative literature is presented in $[24,25]$. It should be noted that the majority of these methods are not really meshless, since they need to use a background mesh for the numerical integration. However, the finite point method (FPM), the point collocation method (MPC), and the meshless local Petrov-Galerkin (MLPG) method are inherently meshless methods.

Herein, the meshless point collocation method is used for the numerical solution of the coupled transient MHD flow in a straight duct having a rectangular, a triangular and a circular cross section, respectively. To the authors' knowledge a very limited number of research works using meshless strongform collocation methods for solving MHD flow problems exists. Namely, the authors in [25] used the meshless point collocation method, to solve steady state MHD problems for rectangular, circular, elliptical and irregular cross section for high Hartmann numbers up to $M=100,000$. element free-Galerkin (EFG) method was used in [26] to solve the steady-state MHD flow. The formulation was applied to the study of two-dimensional magnetohydrodynamic flow problems for moderate Hartmann numbers $(M=500)$, and as the authors claimed, the computed results confirm the accuracy and correctness of the proposed formulation. Moreover, various weak-form meshless methods have been applied for the solution of unsteady MHD flows. Authors in [27] used the local boundary integral equation (LBIE) meshless method to obtain the numerical solution of the coupled equations for the velocity and the magnetic field for unsteady MHD flow through a pipe of rectangular and circular sections with nonconducting walls for low Hartmann numbers up to $M=40$. The MLPG method was used in [28] for the numerical solution of the coupled equations in velocity and magnetic field for unsteady MHD flow through a pipe of rectangular section 
having arbitrary conducting walls. Results were presented for different Hartmann numbers up to 40 .

As it is refereed in [29], hitherto, there has been less research devoted to MFree strong-form methods, compared to weak-form descriptions. This may be partly attributed to the fact that the MFree strong-form method may be less stable than the one based on the weak-form, and partly because research was concentrated on the finite element methods (FEM) which use weak-form descriptions and thus, it was a natural step to adopt the weak-form in MFree methods. In the present paper, a strong-form meshless method is used for the unsteady coupled MHD problems for rectangular, triangular and circular cross sections. The solution is obtained using the collocation formulation and the moving least squares (MLS) approximation. Nevertheless, imposing the boundary conditions is not a straightforward procedure. It is due to the fact that the PCM is based on MLS approximations, which are usually approximants and not interpolants. Elimination of this difficulty requires the introduction of additional unknown parameters, such as Lagrange multipliers, however, it can lead to poor conditioning of the matrix equations [26]. In the present article, we use an interpolatory formulation for MLS approximants that allows the direct introduction of boundary conditions, reduces the processing time and improves the condition numbers, as in $[25,26,29]$. The formulation is applied to the study of two-dimensional magnetohydrodynamic flow problems, and the computed results confirm the accuracy and correctness of the proposed formulation. Velocity and induced magnetic field across the section have been calculated and the contour plots at various time levels, for low $(M \leq 100)$, moderate $(100 \leq M \leq 1,000)$, and high Hartmann $(M \geq 1,000)$ numbers, are presented. A variety of wall conductivity conditions are applied when a uniform magnetic field is imposed perpendicular to the flow direction. Regular and irregular nodal distributions are used, ensuring the positivity conditions [30]. The numerical results of the strong-form LMPC method are compared with those obtained using two weak-form meshless methods, that is, the LBIE meshless method and the MLPG method or the analytical solutions, where they are available. Additionally, for the demonstration of the accuracy and efficiency of the LMPC method, we present the error norms $L_{2}, L_{\infty}$, the convergence rate and the run time of the method.

\section{Physical problem, governing equations and boundary conditions}

The unsteady MHD flows are governed by a set of partial differential equations (PDEs), originated from both NavierStokes and Maxwell equations, for the conducting fluids and for the electromagnetic fields, respectively [26,31]. Thus,

$$
\begin{aligned}
\rho \frac{\partial \boldsymbol{u}}{\partial t}+\rho(\boldsymbol{u} \cdot \nabla) \cdot \boldsymbol{u}= & -\nabla p+\mu \nabla^{2} \boldsymbol{u}+\boldsymbol{J} \times \boldsymbol{B} \\
& +\rho_{e} \boldsymbol{E}, \nabla \cdot \boldsymbol{u}=0,
\end{aligned}
$$

$\nabla \times \boldsymbol{E}=0, \quad \nabla \cdot \boldsymbol{E}=\frac{\rho_{e}}{\varepsilon_{0}}$,

$\boldsymbol{J}=\sigma(\boldsymbol{E}+\boldsymbol{u} \times \boldsymbol{B})+\rho_{e} \boldsymbol{u}$,

$\nabla \times \boldsymbol{B}=\mu_{e} \boldsymbol{J}, \quad \nabla \cdot \boldsymbol{B}=0$,

where $\boldsymbol{u}$ is the velocity field, $\mu$ is the coefficient of viscosity of the fluid, $p$ is the pressure, $\boldsymbol{B}$ is the entire magnetic field (external and induced), $\boldsymbol{E}$ is the induced electric field, $\sigma$ is the constant electrical conductivity, $\rho_{e}$ is the electric charge density, $\varepsilon_{0}$ is the electric permittivity of free space, $\mu_{e}$ is the magnetic permeability and $\rho$ the mass density. The vector $\boldsymbol{J}$ stands for the conduction electric current density.

We consider the unsteady, laminar flow of a viscous, incompressible and electrically conducting fluid driven by a constant applied pressure gradient in a duct having a boundary described by the parametric expression

$f(x, y)=0$.

To specify the problem, it is supposed that the velocity is zero at rigid boundaries, while the fluid motion is fully developed (the duct is assumed to be of infinite length) and the sides of the duct are electrically insulated or having variable conductivity. Furthermore, the analysis will be restricted to a plane which is perpendicular to the direction of the fluid stream. The axis of the duct is chosen as the $z$-direction. Thus, by convention the constant external magnetic field $\boldsymbol{B}_{0}$ is imposed along a random vector having an angle $\varphi$ with the $y$-axis. It is also assumed that all physical quantities (except the pressure $p$ ) are independent of $z$-directional coordinates. So, there is only one component $V_{z}(x, y, t)$ of the velocity field and one component $B_{z}(x, y, t)$ of the induced magnetic field in the $z$-direction and there is no net current flow in the $z$-direction. Thus, the $z$ components of the governing equations becomes

$$
\begin{aligned}
& \mu \nabla^{2} V_{z}+\frac{\left(B_{0}\right)_{x}}{\mu_{e}} \frac{\partial B_{z}}{\partial x}+\frac{\left(B_{0}\right)_{y}}{\mu_{e}} \frac{\partial B_{z}}{\partial y}=\frac{\partial p}{\partial z}+\rho \frac{\partial V_{z}}{\partial t}, \\
& \nabla^{2} B_{z}+\sigma \mu_{e}\left(B_{0}\right)_{x} \frac{\partial V_{z}}{\partial x}+\sigma \mu_{e}\left(B_{0}\right)_{y} \frac{\partial V_{z}}{\partial y}=\frac{\rho}{\mu} \frac{\partial B_{z}}{\partial t} .
\end{aligned}
$$

Thus, the partial differential equations in non-dimensional form in terms of velocity $V(x, y, t)$ and the induced magnetic field $B(x, y, t)$ are

$$
\begin{aligned}
& \nabla^{2} V+M_{x} \frac{\partial B}{\partial x}+M_{y} \frac{\partial B}{\partial y}=-1+\frac{\partial V}{\partial t}, \\
& \nabla^{2} B+M_{x} \frac{\partial V}{\partial x}+M_{y} \frac{\partial V}{\partial y}=\frac{\partial B}{\partial t},
\end{aligned}
$$

where the non-dimensionalization was performed with a characteristic length $L_{0}$ and a characteristic velocity $V_{0}$ 
(mean axis velocity). The dimensionless variables are

$x($ dimensionless $)=\frac{x}{L_{0}}, \quad y($ dimensionless $)=\frac{y}{L_{0}}$,

$V=\frac{V_{z}}{V_{0}}, \quad B=\frac{(\sigma \mu)^{-\frac{1}{2}} B_{z}}{V_{0} \mu_{e}}$,

$V_{0}=\frac{-L_{0}^{2} \frac{\partial p}{\partial z}}{\mu}, \quad t($ dimensionless $)=\frac{t \mu}{L_{0}^{2} \rho}$

and $M$ is the Hartmann number given by

$M=\frac{B_{0} L_{0} \sqrt{\sigma}}{\sqrt{\mu}}$.

Regarding the boundary conditions, having variable electrical conductivity on the walls of the duct means that we have mixed-type boundary conditions for the magnetic field, giving

$\frac{\partial B}{\partial n}+\lambda B=0$

on the boundary of the flow region $\partial \Omega$ in dimensionless form. Here $\lambda=\sigma L_{0} / \sigma^{\prime} h$, where $\sigma^{\prime}$ is the electric conductivity at the walls, $h$ is the thickness of the walls which is assumed to be small and $\boldsymbol{n}$ is the outward normal direction. When $\lambda=0$, the walls are perfectly conducting and as $\lambda \rightarrow \infty$ the walls are considered insulated. The initial conditions are

$V(x, y, 0)=0, \quad B(x, y, 0)=0, \quad(x, y) \in \Omega$.

\section{Moving least squares approximation}

Numerous techniques in the area of the meshless methods have been developed in order to construct shape functions [32]. The most widely used methods are the MLS approximation [33] and the radial point interpolation method (RPIM) [34]. Among these methods, the MLS one has been widely used for function approximation. The advantages of MLS can be summarized as follows; firstly, there is no need for predefined mesh connectivity during the construction of MLS shape functions. Secondly, the high-order continuity of shape functions eliminates the necessity of solving the weak-forms of governing equations; and, finally, the availability of smooth derivatives eliminates the need for costly procedure of gradient approximation and recovery. Even though, there is an alternative way to generate derivatives of shape functions for the point collocation scheme $[35,36]$, the classical methodology, presented in [32], was adopted.

\subsection{Methodology}

In the moving least-squares technique, the approximation $u^{h}(\boldsymbol{x})$ is expressed as the inner product of a vector of the linearly independent polynomial basis, $\boldsymbol{p}(\boldsymbol{x})$ and a vector of the unknown coefficients, $\boldsymbol{a}(\boldsymbol{x})$, to be determined by the approximation algorithm

$u^{h}(\boldsymbol{x})=\sum_{i=1}^{m} p_{i}(\boldsymbol{x}) \alpha_{i}(\boldsymbol{x}) \equiv \boldsymbol{p}^{\boldsymbol{T}}(\boldsymbol{x}) \boldsymbol{a}(\boldsymbol{x})$,

where $\boldsymbol{p}(\boldsymbol{x}) \in \mathbb{R}^{m}, \boldsymbol{a}(\boldsymbol{x}) \in \mathbb{R}^{m}$ and $m$ is the number of monomials in the polynomial basis (We use the polynomial basis of second order). The polynomial basis of order $m$ in one and two dimensions are given by

$$
\begin{aligned}
\boldsymbol{p}^{\boldsymbol{T}}(\boldsymbol{x}) & =\left[1, x, x^{2}, \ldots, x^{m}\right], \\
\boldsymbol{p}^{\boldsymbol{T}}(\boldsymbol{x}) & =\boldsymbol{p}^{\boldsymbol{T}}(x, y) \\
& =\left[1, x, y, x^{2}, x y, y^{2}, \ldots, x^{m}, \ldots, x y^{m-1}, y^{m}\right] .
\end{aligned}
$$

Herein, we use a second order $(m=2)$ polynomial basis, obtaining

$$
\begin{aligned}
\boldsymbol{p}^{\boldsymbol{T}}(\boldsymbol{x})= & {\left[1,\left(x-x_{i}\right),\left(y-y_{i}\right),\left(x-x_{i}\right)^{2},\right.} \\
& \left.\left(x-x_{i}\right)\left(y-y_{i}\right),\left(y-y_{i}\right)^{2}\right] .
\end{aligned}
$$

The local character of the MLS approximation can be viewed as a generalization of the traditional least-squares approximation in which the vector $\boldsymbol{a}$ is not a function of $\boldsymbol{x}$. Equation (12) is referred to as the global least-squares approximation. In addition, there exists a unique local approximation associated with each point in the domain. In order to determine the form of $\boldsymbol{a}(\boldsymbol{x})$, a weighted discrete error norm,

$J(\boldsymbol{x})=\sum_{I=1}^{n} w_{I}(\boldsymbol{x})\left[\sum_{j=1}^{m} p_{j}^{\mathrm{T}}\left(\boldsymbol{x}_{I}\right) a(\boldsymbol{x})-u_{I}\right]^{2}$

is constructed and minimized. Here, $w_{I}(\boldsymbol{x}) \equiv w\left(\boldsymbol{x}-\boldsymbol{x}_{I}\right)$ denotes the weight function associated with node $I$, and the quantity in brackets is the difference between the local approximation at node $I$ and the data at node $I, u_{I}$, and $n$ is the number of nodes in the support domain of $w_{I}(\boldsymbol{x})$. The minimization of Eq. (16) with respect to $\boldsymbol{a}(\boldsymbol{x})$ determines $\boldsymbol{a}(\boldsymbol{x})$. The local approximation associated with point $\boldsymbol{x}$ is only used in the minimization process, and is equivalent to the global approximation at the single point $\boldsymbol{x}$. Compact support of the weight functions provides the local character the moving least-squares method.

\subsection{Shape functions and their derivatives}

The minimization of Eq. (16),

$\frac{\partial J(\boldsymbol{x})}{\partial \boldsymbol{a}(\boldsymbol{x})}=0$

results in the linear system

$\boldsymbol{A}(\boldsymbol{x}) \boldsymbol{a}(\boldsymbol{x})=\boldsymbol{B}(\boldsymbol{x}) \boldsymbol{U}_{S}$, 
Fig. 1 a Spatial domain and nodal distribution, b support domain illustration

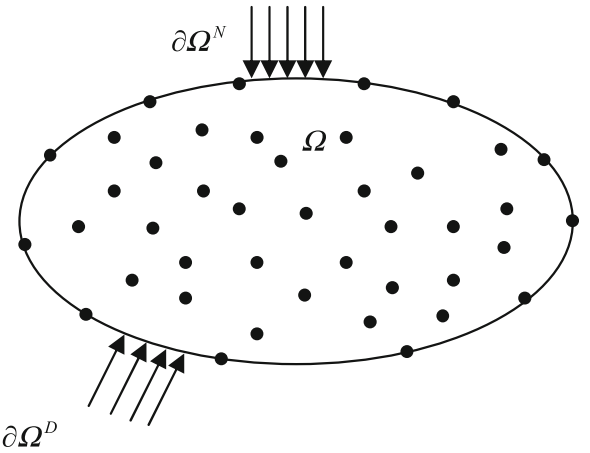

(a)

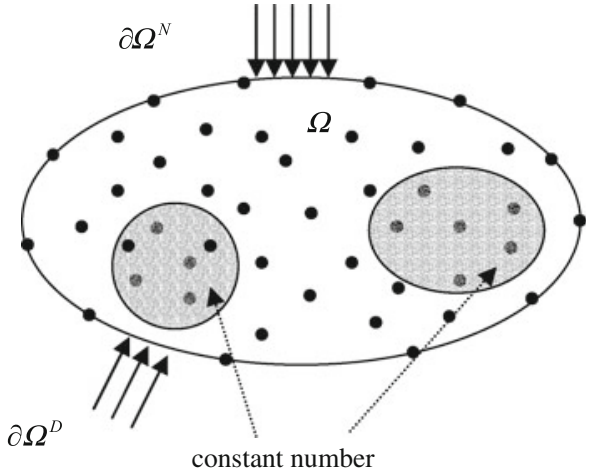

(b) where $\boldsymbol{U}_{s}$ is a vector containing the nodal data, $\boldsymbol{U}_{s}^{\mathrm{T}}=$ $\left[u_{1}, u_{2}, \ldots, u_{n}\right]$, and

$$
\begin{aligned}
\boldsymbol{A}(\boldsymbol{x}) & =\sum_{I=1}^{n} w_{I}(\boldsymbol{x}) \boldsymbol{p}\left(\boldsymbol{x}_{I}\right) \boldsymbol{p}^{\mathrm{T}}\left(\boldsymbol{x}_{I}\right), \\
\boldsymbol{B}(\boldsymbol{x}) & =\left[w_{1}(\boldsymbol{x}) \boldsymbol{p}\left(\boldsymbol{x}_{1}\right) w_{2}(\boldsymbol{x}) \boldsymbol{p}\left(\boldsymbol{x}_{2}\right) \cdots w_{n}(\boldsymbol{x}) \boldsymbol{p}\left(\boldsymbol{x}_{n}\right)\right],
\end{aligned}
$$

where $\boldsymbol{A} \in \mathbb{R}^{\boldsymbol{m} \times \boldsymbol{m}}$ and $\boldsymbol{B} \in \mathbb{R}^{\boldsymbol{m} \times \boldsymbol{n}}$. The matrix $\boldsymbol{A}$ must be inverted at every sampling point. Substitution of the solution of Eq. (18) into the global approximation (Eq. (12)), completes the least-squares approximation.

$u^{h}(\boldsymbol{x})=\underbrace{\boldsymbol{p}^{\mathrm{T}}(\boldsymbol{x}) \boldsymbol{A}^{-1}(\boldsymbol{x}) \boldsymbol{B}(\boldsymbol{x})}_{\boldsymbol{\phi}(x)} \boldsymbol{U}_{s}$.

Here, the spatial dependence has been lumped into one row matrix, $\phi(x)$, and the approximation therefore takes the form of a product of a matrix of shape functions with a vector of nodal data. Derivatives of the shape functions may be calculated by applying the product rule to

$\phi=p^{\mathrm{T}} \boldsymbol{A}^{-1} \boldsymbol{B}$.

In order to obtain the spatial derivatives of the approximation function $u^{h}(\boldsymbol{x})$, it is necessary to obtain the derivatives of the MLS shape functions $\phi_{i}(\boldsymbol{x})$,

$$
\begin{aligned}
\frac{\partial}{\partial x_{j}} u^{h}(\boldsymbol{x}) & =\frac{\partial}{\partial x_{j}} \sum_{i=1}^{n} \phi_{i}(\boldsymbol{x}) u_{i} \\
& =\sum_{i=1}^{n}\left\{\frac{\partial}{\partial x_{j}} \phi_{i}(\boldsymbol{x})\right\} u_{i}, \quad x_{j}=x, y, z .
\end{aligned}
$$

The derivative of the shape function is given as

$$
\begin{aligned}
\frac{\partial \boldsymbol{\phi}(\boldsymbol{x})}{\partial x_{j}}= & \frac{\partial\left(\boldsymbol{p}^{\mathrm{T}} \boldsymbol{A}^{-1} \boldsymbol{B}_{i}\right)}{\partial x_{j}}=\frac{\partial \boldsymbol{p}^{\mathrm{T}}}{\partial x_{j}} \boldsymbol{A}^{-1} \boldsymbol{B}_{i}+\boldsymbol{p}^{\mathrm{T}} \frac{\partial\left(\boldsymbol{A}^{-1}\right)}{\partial x_{j}} \boldsymbol{B}_{i} \\
& +\boldsymbol{p}^{\mathrm{T}} \boldsymbol{A}^{-1} \frac{\partial \boldsymbol{B}_{i}}{\partial x_{j}}, \quad x_{j}=x, y, z
\end{aligned}
$$

where $\frac{\partial\left(\boldsymbol{A}^{-1}\right)}{\partial x_{j}}=-\boldsymbol{A}^{-1}(\boldsymbol{x}) \frac{\partial \boldsymbol{A}(\boldsymbol{x})}{\partial x_{j}} \boldsymbol{A}^{-1}(\boldsymbol{x})$. Regarding the second order derivative of the unknown function we get

$$
\begin{aligned}
\frac{\partial^{2} \boldsymbol{\phi}(x)}{\partial x_{j}^{2}}= & \frac{\partial}{\partial x_{j}}\left(\frac{\partial \boldsymbol{\phi}(\boldsymbol{x})}{\partial x_{j}}\right) \\
= & \frac{\partial}{\partial x_{j}}\left(\frac{\partial \boldsymbol{p}^{\mathrm{T}}}{\partial x_{j}} \boldsymbol{A}^{-1} \boldsymbol{B}_{i}+\boldsymbol{p}^{\mathrm{T}} \frac{\partial\left(\boldsymbol{A}^{-1}\right)}{\partial x_{j}} \boldsymbol{B}_{i}+\boldsymbol{p}^{\mathrm{T}} \boldsymbol{A}^{-1} \frac{\partial \boldsymbol{B}_{i}}{\partial x_{j}}\right) \\
= & \frac{\partial^{2} \boldsymbol{p}^{\mathrm{T}}}{\partial x_{j}^{2}} \boldsymbol{A}^{-1} \boldsymbol{B}_{i}+\frac{\partial \boldsymbol{p}^{\mathrm{T}}}{\partial x_{j}} \frac{\partial\left(\boldsymbol{A}^{-1}\right)}{\partial x_{j}} \boldsymbol{B}_{i}+\frac{\partial \boldsymbol{p}^{\mathrm{T}}}{\partial x_{j}} \boldsymbol{A}^{-1} \frac{\partial \boldsymbol{B}_{i}}{\partial x_{j}} \\
& +\frac{\partial \boldsymbol{p}^{\mathrm{T}}}{\partial x_{j}} \frac{\partial\left(\boldsymbol{A}^{-1}\right)}{\partial x_{j}} \boldsymbol{B}_{i}+\boldsymbol{p}^{\mathrm{T}} \frac{\partial^{2}\left(\boldsymbol{A}^{-1}\right)}{\partial x_{j}^{2}} \boldsymbol{B}_{i}+\boldsymbol{p}^{\mathrm{T}} \frac{\partial\left(\boldsymbol{A}^{-1}\right)}{\partial x_{j}} \frac{\partial B_{i}}{\partial x_{j}} \\
& +\frac{\partial \boldsymbol{p}^{\mathrm{T}}}{\partial x_{j}} \boldsymbol{A}^{-1} \frac{\partial \boldsymbol{B}_{i}}{\partial x_{j}}+\boldsymbol{p}^{\mathrm{T}} \frac{\partial\left(\boldsymbol{A}^{-1}\right)}{\partial x_{j}} \frac{\partial \boldsymbol{B}_{i}}{\partial x_{j}}+\boldsymbol{p}^{\mathrm{T}} \boldsymbol{A}^{-1} \frac{\partial^{2} \boldsymbol{B}_{i}}{\partial x_{j}^{2}},
\end{aligned}
$$

where $x_{j}=x, y, z$ and $\frac{\partial^{2}\left(\boldsymbol{A}^{-1}\right)}{\partial x_{j}^{2}}=-\frac{\partial\left(\boldsymbol{A}^{-1}\right)}{\partial x_{j}} \boldsymbol{A} \boldsymbol{A}^{-1}-\boldsymbol{A}^{-1} \frac{\partial \boldsymbol{A}}{\partial x_{j}}$ $\boldsymbol{A}^{-1}-\boldsymbol{A}^{-1} \boldsymbol{A} \frac{\partial\left(\boldsymbol{A}^{-1}\right)}{\partial x_{j}}$.

\subsection{Weight function}

The weight function is non-zero over a small neighborhood of $\boldsymbol{x}_{i}$, called the support domain of node $i$. The choice of the weight function $W\left(\boldsymbol{x}-\boldsymbol{x}_{i}\right)$ affects the resulting approximation $u^{h}\left(\boldsymbol{x}_{i}\right)$ significantly. In the present paper a Gaussian weight function is used [32,37], yet the support domain does not have a standard point density value. Instead, a constant number of nodes are used for the approximation of the field function, Fig. 1.

$W\left(\boldsymbol{x}-\boldsymbol{x}_{i}\right) \equiv W(d)=\left\{\begin{array}{l}e^{-\left(\frac{d_{I}}{a}\right)^{2}} \\ 0\end{array}\right\}$,

where $I=1,2,3, \ldots, q$ are the nodes that produce the support domain of node $\boldsymbol{x}_{i}$, and $d=\frac{\left|\boldsymbol{x}-\boldsymbol{x}_{i}\right|}{a_{0}^{2}}$ with $a_{0}$ a prescribed constant (often $a_{0}=0.2$ ). 


\section{Meshless point collocation method}

The meshless point collocation method is a meshless "strongform" description one. The "strong-form" description of the governing equations and boundary conditions is used and discretized by collocation techniques. The aforementioned formulations possess the following attractive advantages. They are truly meshless and the implementing procedure is straightforward, while the algorithms and the implementation can be kept simple, particularly when handling problems with Dirichlet boundary conditions [32]. Under these conditions, strong-form methods are highly efficient computationally, even with polynomial approximation functions, and the solution can be systematically obtained with increased accuracy, compared to FEM, FDM, or other computational methods. In general, MFree strong-form methods may still suffer from some local stability and accuracy issues, depending on the problem [32]. However, these local restrictions are now systematically avoided with the utilization of TypeI nodal distribution and proper local point cloud refinement procedures, in accordance with [25], even for natural or mixed type boundary conditions.

In this section we present collocation scheme using the MLS approximation to spatially discretize the unsteady homogeneous diffusion equation. We also present a $\theta$-weighted time-stepping scheme for temporal discretization. The use of an interpolatory formulation for MLS approximants, allows the direct introduction of boundary conditions, reduces the processing time and improves thus the condition numbers.

Consider the governing equations of the unsteady problem

$\nabla^{2} V+M_{x} \frac{\partial B}{\partial x}+M_{y} \frac{\partial B}{\partial y}=-f_{1}+\frac{\partial V}{\partial t}$,

$\nabla^{2} B+M_{x} \frac{\partial V}{\partial x}+M_{y} \frac{\partial V}{\partial y}=f_{2}+\frac{\partial B}{\partial t}$,

where $f_{1}=1$ and $f_{2}=0$. We use these symbols for the sake of generalization. The boundary conditions are

$\frac{\partial B}{\partial n}+\lambda B=0, \quad V=0, \quad(x, y) \in \partial \Omega$
By the MLS approximation we get $u(\boldsymbol{x}, t)=\sum_{i=1}^{N} \Phi_{i}(\boldsymbol{x})$ $u_{i}(t) \equiv \boldsymbol{\Phi} \boldsymbol{U}_{\boldsymbol{s}}$ for the unknown function, $u_{q}(\boldsymbol{x}, t)=$ $\sum_{i=1}^{N} \frac{\partial \Phi_{i}(\boldsymbol{x})}{\partial q} u_{i}(t) \equiv \boldsymbol{\Phi}_{\boldsymbol{q}} \boldsymbol{U}_{\boldsymbol{s}}$ for the partial $x, y$ derivative, and $u_{q q}(\boldsymbol{x}, t)=\sum_{i=1}^{N} \frac{\partial^{2} \Phi_{i}(x)}{\partial \boldsymbol{q}^{2}} u_{i}(t) \equiv \boldsymbol{\Phi}_{\boldsymbol{q} \boldsymbol{q}} \boldsymbol{U}_{\boldsymbol{s}}$ for the second $x, y$ partial derivative. Additionally, we set $n_{d}$ as the number of nodes in the interior and $n_{b}$ as the number of nodes on the boundary and the total number of nodes as $N\left(N=n_{d}+n_{b}\right)$.

The first equation, Eq. (27), can be written as

$\frac{\partial V}{\partial t}+\left(-\nabla^{2} V-M_{x} \frac{\partial B}{\partial x}-M_{y} \frac{\partial B}{\partial y}\right)=1$.

From the notation described above and using the Euler's $\theta$-weighted time-stepping scheme for temporal discretization, for the interior nodes we get

$$
\begin{aligned}
& \boldsymbol{\Phi}_{\boldsymbol{d}} \frac{\boldsymbol{V}^{n+1}-\boldsymbol{V}^{n}}{\delta t}+\theta\left(-\left(\boldsymbol{\Phi}_{\boldsymbol{d}, \boldsymbol{x} \boldsymbol{x}}+\boldsymbol{\Phi}_{\boldsymbol{d}, \boldsymbol{y} \boldsymbol{y}}\right) \boldsymbol{V}^{n+1}\right. \\
& \left.-M_{x} \boldsymbol{\Phi}_{\boldsymbol{d}, \boldsymbol{x}} \boldsymbol{B}^{n+1}-M_{y} \boldsymbol{\Phi}_{\boldsymbol{d}, \boldsymbol{y}} \boldsymbol{B}^{n+1}\right) \\
& \quad+(1-\theta)\left(-\left(\boldsymbol{\Phi}_{\boldsymbol{d}, \boldsymbol{x} \boldsymbol{x}}+\Phi_{\boldsymbol{d}, \boldsymbol{y} \boldsymbol{y}}\right) \boldsymbol{V}^{n}-M_{x} \boldsymbol{\Phi}_{\boldsymbol{d}, \boldsymbol{x}} \boldsymbol{B}^{n}\right. \\
& \left.\quad-M_{y} \boldsymbol{\Phi}_{\boldsymbol{d}, \boldsymbol{y}} \boldsymbol{B}^{n}\right)=\boldsymbol{f}^{n+1} .
\end{aligned}
$$

Multiplying both parts with $\delta t$ we can write

$$
\begin{aligned}
& \boldsymbol{\Phi}_{\boldsymbol{d}} \boldsymbol{V}^{n+1}-\boldsymbol{\Phi}_{\boldsymbol{d}} \boldsymbol{V}^{n}+\theta \delta t\left(-\left(\boldsymbol{\Phi}_{\boldsymbol{d}, \boldsymbol{x} x}+\boldsymbol{\Phi}_{\boldsymbol{d}, \boldsymbol{y} \boldsymbol{y}}\right) \boldsymbol{V}^{n+1}\right) \\
& +\theta \delta t\left(-M_{x} \boldsymbol{\Phi}_{\boldsymbol{d}, \boldsymbol{x}} \boldsymbol{B}^{n+1}\right)+\theta \delta t\left(-M_{y} \boldsymbol{\Phi}_{\boldsymbol{d}, \boldsymbol{y}} \boldsymbol{B}^{n+1}\right) \\
& +(1-\theta) \delta t\left(-\left(\boldsymbol{\Phi}_{\boldsymbol{d}, \boldsymbol{x} \boldsymbol{x}}+\boldsymbol{\Phi}_{\boldsymbol{d}, \boldsymbol{y} \boldsymbol{y}}\right) \boldsymbol{V}^{n}\right) \\
& +(1-\theta) \delta t\left(-M_{x} \boldsymbol{\Phi}_{\boldsymbol{d}, \boldsymbol{x}} \boldsymbol{B}^{n}\right)+(1-\theta) \delta t\left(-M_{y} \boldsymbol{\Phi}_{\boldsymbol{d}, \boldsymbol{y}} \boldsymbol{B}^{n}\right) \\
& =\delta t \boldsymbol{f}^{n+1}
\end{aligned}
$$

and as matrix notation for the interior nodes $n_{d}$ and incorporating the boundary conditions at $n_{b}$ boundary nodes we have

$$
\begin{aligned}
& {\left[\begin{array}{lll}
\boldsymbol{\Phi}_{\boldsymbol{d}}+\theta \delta t\left(-\left(\boldsymbol{\Phi}_{\boldsymbol{d}, \boldsymbol{x} \boldsymbol{x}}+\boldsymbol{\Phi}_{\boldsymbol{d}, \boldsymbol{y} \boldsymbol{y}}\right)\right) & \theta \delta t\left(-M_{x} \boldsymbol{\Phi}_{\boldsymbol{d}, \boldsymbol{x}}\right)+\theta \delta t\left(-M_{y} \boldsymbol{\Phi}_{\boldsymbol{d}, \boldsymbol{y}}\right) \\
G_{V} \boldsymbol{\Phi}_{b} & 0
\end{array}\right]\left[\begin{array}{l}
\boldsymbol{V}_{N}^{n+1} \\
\boldsymbol{B}_{N}^{n+1}
\end{array}\right]} \\
& \quad=\left[\begin{array}{ll}
\boldsymbol{\Phi}-(1-\theta) \delta t\left(-\left(\boldsymbol{\Phi}_{\boldsymbol{d}, \boldsymbol{x} \boldsymbol{x}}+\boldsymbol{\Phi}_{\boldsymbol{d}, \boldsymbol{y} \boldsymbol{y}}\right)\right) & -(1-\theta) \delta t\left(-M_{x} \boldsymbol{\Phi}_{\boldsymbol{d}, \boldsymbol{x}}\right)-(1-\theta) \delta t\left(-M_{y} \boldsymbol{\Phi}_{\boldsymbol{d}, \boldsymbol{y}}\right) \\
\mathbf{0} & \mathbf{0}
\end{array}\right]\left[\begin{array}{c}
\boldsymbol{V}_{N}^{n} \\
\boldsymbol{B}_{N}^{n}
\end{array}\right]+\left[\begin{array}{l}
\delta \boldsymbol{t} \boldsymbol{f}_{1}^{n+1} \\
\boldsymbol{g}_{1}^{n+1}
\end{array}\right],
\end{aligned}
$$

on the boundary of the flow region and, the initial conditions are

$V(x, y, 0)=0, \quad B(x, y, 0)=0, \quad(x, y) \in \Omega$. where $G_{V}$ is the operator defining the boundary conditions. These equations can be written in a more compact manner by setting 


$$
\begin{aligned}
\boldsymbol{H}_{\boldsymbol{A}}^{+} & =\left[\begin{array}{ll}
\boldsymbol{\Phi}_{\boldsymbol{d}}+\theta \delta t\left(-\left(\boldsymbol{\Phi}_{\boldsymbol{d}, \boldsymbol{x} \boldsymbol{x}}+\boldsymbol{\Phi}_{\boldsymbol{d}, \boldsymbol{y} \boldsymbol{y}}\right)\right) & \theta \delta t\left(-M_{x} \boldsymbol{\Phi}_{\boldsymbol{d}, \boldsymbol{x}}\right)+\theta \delta t\left(-M_{y} \boldsymbol{\Phi}_{\boldsymbol{d}, \boldsymbol{y}}\right) \\
G_{V} \boldsymbol{\Phi}_{\boldsymbol{b}} & \mathbf{0}
\end{array}\right], \\
\boldsymbol{H}_{\boldsymbol{A}}^{-} & =\left[\begin{array}{ll}
\boldsymbol{\Phi}_{\boldsymbol{d}}-(1-\theta) \delta t\left(-\left(\boldsymbol{\Phi}_{\boldsymbol{d}, \boldsymbol{x} \boldsymbol{x}}+\boldsymbol{\Phi}_{\boldsymbol{d}, \boldsymbol{y} \boldsymbol{y}}\right)\right) & -(1-\theta) \delta t\left(-M_{x} \boldsymbol{\Phi}_{\boldsymbol{d}, \boldsymbol{x}}\right)-(1-\theta) \delta t\left(-M_{y} \boldsymbol{\Phi}_{\boldsymbol{d}, \boldsymbol{y}}\right) \\
\mathbf{0} & \mathbf{0}
\end{array}\right], \\
\boldsymbol{F}_{\boldsymbol{A}} & =\left[\begin{array}{l}
\delta t \boldsymbol{f}_{1}^{n+1} \\
\boldsymbol{g}_{1}^{n+1}
\end{array}\right],
\end{aligned}
$$

$\underset{\mathbf{0} \in \mathbb{R}^{\boldsymbol{n}_{\boldsymbol{d}} \times 1}}{\text { where } \boldsymbol{H}_{\boldsymbol{A}}^{+}} \in \mathbb{R}^{\boldsymbol{N} \times 2 \boldsymbol{N}}, \boldsymbol{H}_{\boldsymbol{A}}^{-} \in \mathbb{R}^{\boldsymbol{N} \times 2 \boldsymbol{N}}, \boldsymbol{F}_{\boldsymbol{A}} \in \mathbb{R}^{\boldsymbol{N} \times 1}$ and

Using the same procedure for Eq. (28) we obtain (in matrix notation)

$$
\begin{aligned}
\boldsymbol{H}_{\boldsymbol{B}}^{+} & =\left[\begin{array}{ll}
\theta \delta t\left(-M_{x} \boldsymbol{\Phi}_{\boldsymbol{d}, \boldsymbol{x}}\right)+\theta \delta t\left(-M_{y} \boldsymbol{\Phi}_{\boldsymbol{d}, \boldsymbol{y}}\right) & \boldsymbol{\Phi}_{\boldsymbol{d}}+\theta \delta t\left(-\left(\boldsymbol{\Phi}_{\boldsymbol{d}, \boldsymbol{x} \boldsymbol{x}}+\boldsymbol{\Phi}_{\boldsymbol{d}, \boldsymbol{y} \boldsymbol{y}}\right)\right) \\
\mathbf{0} & G_{B} \boldsymbol{\Phi}_{\boldsymbol{b}}
\end{array}\right], \\
\boldsymbol{H}_{\boldsymbol{B}}^{-} & =\left[\begin{array}{ll}
-(1-\theta) \delta t\left(-M_{x} \boldsymbol{\Phi}_{\boldsymbol{d}, \boldsymbol{x}}\right)-(1-\theta) \delta t\left(-M_{y} \boldsymbol{\Phi}_{\boldsymbol{d}, \boldsymbol{y}}\right) & \boldsymbol{\Phi}_{\boldsymbol{d}}-(1-\theta) \delta t\left(-\left(\boldsymbol{\Phi}_{\boldsymbol{d}, \boldsymbol{x} \boldsymbol{x}}+\boldsymbol{\Phi}_{\boldsymbol{d}, \boldsymbol{y} \boldsymbol{y}}\right)\right)
\end{array}\right], \\
\boldsymbol{F}_{\boldsymbol{B}} & =\left[\begin{array}{l}
\delta t \boldsymbol{f}_{2}^{n+1} \\
\boldsymbol{g}_{2}^{n+1}
\end{array}\right],
\end{aligned}
$$

where $\boldsymbol{H}_{\boldsymbol{B}}^{+} \in \mathbb{R}^{N \times 2 N}, \boldsymbol{H}_{B}^{-} \in \mathbb{R}^{N \times 2 N}$ and $\boldsymbol{F}_{\boldsymbol{B}} \in \mathbb{R}^{N \times 1}$.

The final system of the uncoupled partial differential equations of the MHD flow can be obtained as

$$
\left[\begin{array}{l}
\boldsymbol{H}_{\boldsymbol{A}}^{+} \\
\boldsymbol{H}_{\boldsymbol{B}}^{+}
\end{array}\right]\left[\begin{array}{l}
\boldsymbol{V}^{n+1} \\
\boldsymbol{B}^{n+1}
\end{array}\right]=\left[\begin{array}{l}
\boldsymbol{H}_{\boldsymbol{A}}^{-} \\
\boldsymbol{H}_{\boldsymbol{B}}^{-}
\end{array}\right]\left[\begin{array}{l}
\boldsymbol{V}^{n} \\
\boldsymbol{B}^{n}
\end{array}\right]+\left[\begin{array}{l}
\boldsymbol{F}_{\boldsymbol{A}} \\
\boldsymbol{F}_{\boldsymbol{B}}
\end{array}\right]
$$

Finally, setting

$$
\begin{aligned}
& \boldsymbol{u}^{n}=\left[\begin{array}{l}
\boldsymbol{V}^{n} \\
\boldsymbol{B}^{n}
\end{array}\right], \quad \boldsymbol{F}=\left[\begin{array}{l}
\boldsymbol{F}_{\boldsymbol{A}} \\
\boldsymbol{F}_{\boldsymbol{B}}
\end{array}\right], \quad Q^{+}=\left[\begin{array}{l}
\boldsymbol{H}_{\boldsymbol{A}}^{+} \\
\boldsymbol{H}_{\boldsymbol{B}}^{+}
\end{array}\right], \\
& \boldsymbol{Q}^{-}=\left[\begin{array}{l}
\boldsymbol{H}_{\boldsymbol{A}}^{-} \\
\boldsymbol{H}_{\boldsymbol{B}}^{-}
\end{array}\right],
\end{aligned}
$$

Eq. (27) then take the form

$$
\boldsymbol{u}^{n+1}=\left(Q^{+}\right)^{-1}\left(Q^{-} \boldsymbol{u}^{n}+\boldsymbol{F}\right)
$$

where $Q^{+} \in \mathbb{R}^{2 N \times 2 N}, Q^{-} \in \mathbb{R}^{2 N \times 2 N}$ and $\boldsymbol{F} \in \mathbb{R}^{2 N \times 1}$.

\section{Numerical results}

5.1 Unsteady MHD flow in a rectangular duct with insulating or conducting walls

As a first numerical example, we examined a laminar, incompressible and electrically conducting fluid in a square duct with variable wall conductivity, with the applied magnetic field along the direction of $x$-axis, as shown in Fig. 2.

The coupled MHD equations, discretized with the meshless point collocation method, are solved using various types of boundary conditions at a square pipe $(|x| \leq 1,|y| \leq 1)$. In order to establish the validity of the numerical results, we first solved the steady state MHD flow problem, followed the unsteady flow case and reaching the steady-state solution as $\tau \rightarrow \infty$ for the time domain $(0, \tau)$. The Type-I nodal distribution of the spatial domain $\Omega$ is used, providing the convergence of the discrete Laplacian operator [38]. Numerical results are presented at different time intervals for selected nodes at small, moderate and high Hartmann numbers. The numerical results were compared with those obtained from others weak-form meshless numerical methods or the analytical solutions.

\section{Case 1: Insulating walls}

In Figs. 3 and 4, the velocity and the induced magnetic field contours are presented at the steady state case for low

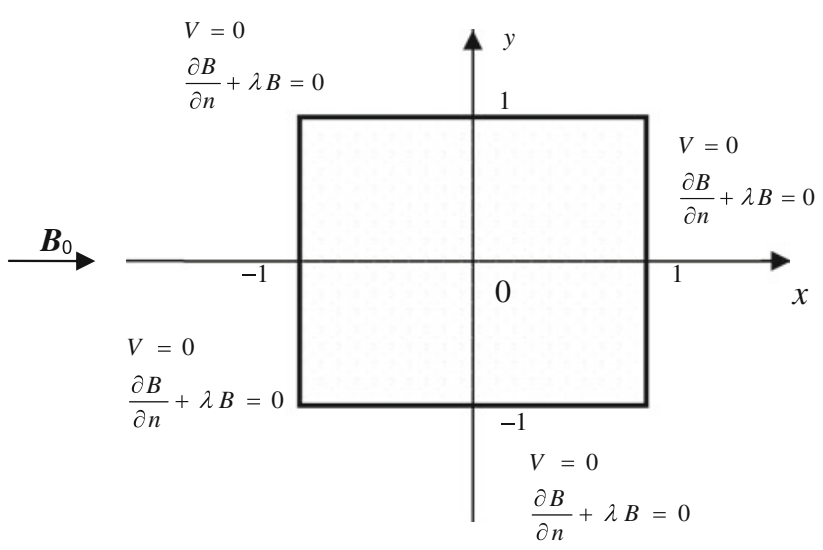

Fig. 2 Square section of the duct with variable wall conductivity 

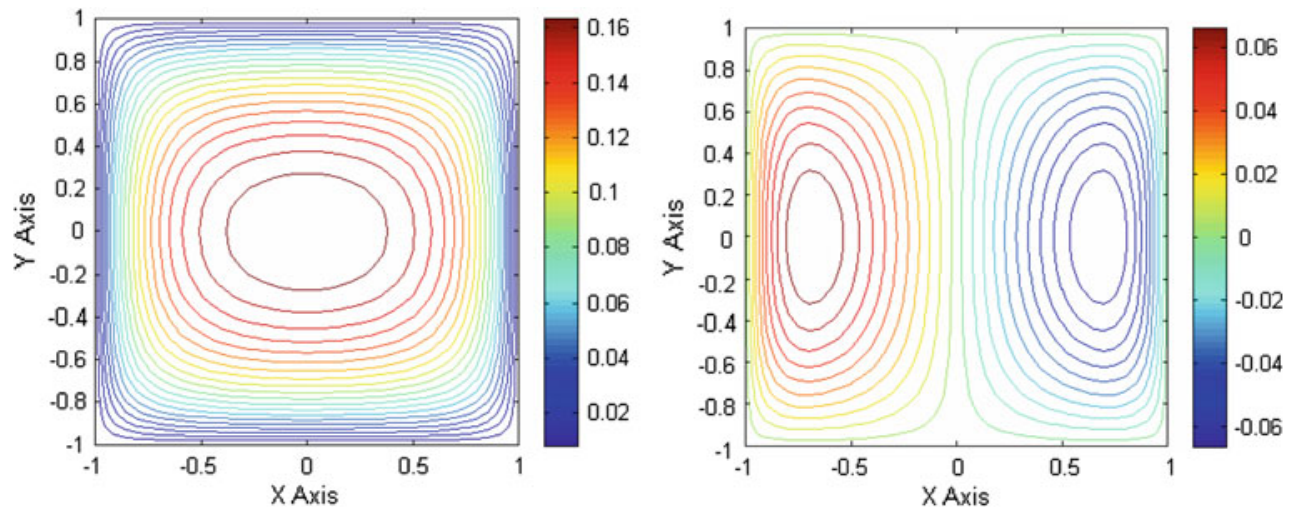

Fig. 3 Contours of velocity and induced magnetic field for $M=5$ and $\lambda \rightarrow \infty$
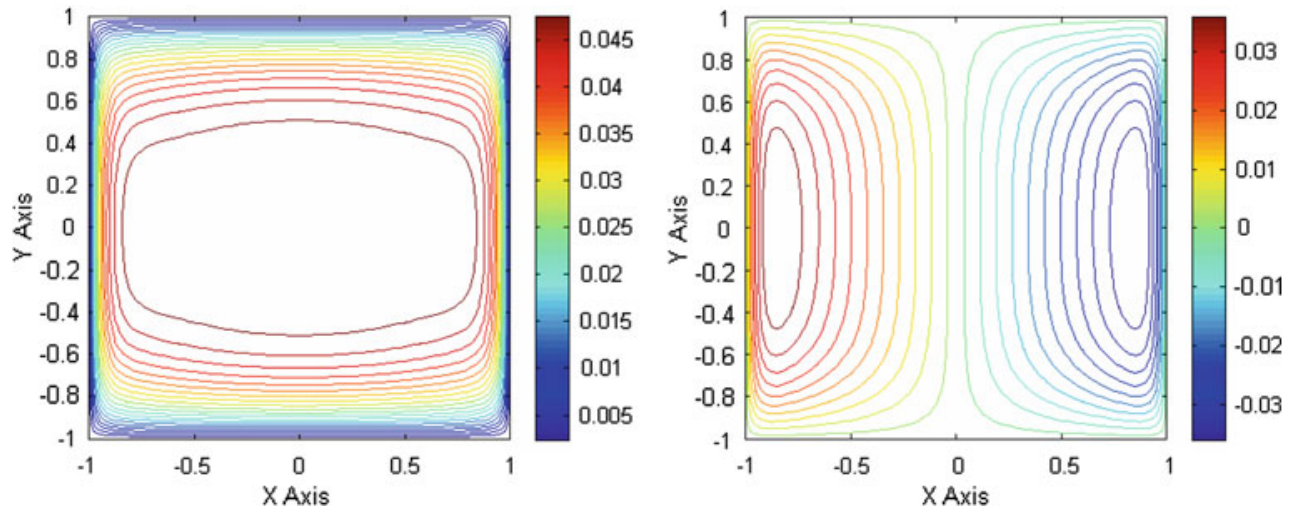

Fig. 4 Contours of velocity and induced magnetic field for $M=20$ and $\lambda \rightarrow \infty$

Hartmann numbers $M=5$ and $M=20$, respectively, for non-conducting walls $(\lambda \rightarrow \infty)$. Furthermore, numerical results are obtained for various time steps. As the Hartmann number increases the time step $\delta t$ needs to obtain smaller values in order to increase the accuracy. Thus, for low Hartmann numbers $(M \leq 50)$ the calculations are performed using a time step $\delta t=0.005$, having the total number of regular distributed nodes $N=961$. On the other hand, for moderate Hartmann numbers $(M \leq 1,000)$ the time step is decreased to $\delta t=0.001$. As the Hartmann number increases, boundary layers are formed and the numerical solutions are very sensitive to the presence of unwanted oscillations. In order to avoid these oscillations the nodal distribution is again regular, with an aspect ratio 2:1, however, since the boundary layers are formed at $x=1$ and $x=-1$. The numerical results are compared with those obtained using the local boundary integral method (LBIE) [27] and the meshless-local PetrovGalerkin (MLPG) method [28]. The comparison reveals that, as time increases the numerical solutions of the proposed scheme for the velocity and the induced magnetic field tend to the exact solution of the steady state problem [3]. More precisely, Tables 1 and 2 present the numerical solutions of velocity for some selected points at different times for $M=5$ and $M=20$, respectively.
For future validation purposes, Table 3 presents the numerical results of the velocity, with LMPCM, for selected points at distinct time values for $M=500$. For high Hartmann numbers the solution reach the steady state in a small period of time, and numerical results are presented for times values near the initial one $t=0$. Results obtained with FEM in [18] are also presented.

As the Hartmann number is increased, $M=1,000$ and $M=10,000$ (Fig. 5), the velocity of the flow becomes more uniform throughout the region. However, at a very narrow part of the domain, adjacent to the walls, boundary layers are formed, initially near the boundaries $x=1$ and $x=-1$, (Figs. 3, 4) and finally, next to all of the boundaries (Fig. 5). A layer formation is also observed for the induced magnetic field, emanating from the boundaries $x=1$ and $x=-1$. The layers of the insulated walls are of order $O(1 / M)$ [31].

\section{Case 2: Conducting walls}

Now, we consider the unsteady MHD flow in a duct with arbitrary conductivity on the walls of the duct. A constant variation is assumed for each time step and the problems is solved on the time domain $(0, \tau)$ as $\tau \rightarrow \infty$ up to Hartman numbers $M=300$ and $\lambda=300$. Figure 6 shows the 
Table 1 Numerical solution of velocity for some selected points at different times for non-conducting walls and $M=5$

\begin{tabular}{|c|c|c|c|c|c|c|c|}
\hline$(\mathrm{x}, \mathrm{y})$ & Numerical method & $t=0.1$ & $t=0.2$ & $t=0.5$ & $t=1.0$ & & Steady state $(=$ exact $)$ \\
\hline \multirow[t]{3}{*}{$(0.00,0.00)$} & LMPC & 0.09050 & 0.14346 & 0.17060 & 0.17159 & $\rightarrow$ & 0.17160 \\
\hline & LBIE & 0.09366 & 0.14398 & 0.17009 & 0.17105 & $\rightarrow$ & 0.17160 \\
\hline & MLPG & 0.0944 & 0.1450 & 0.1708 & N/A & $\rightarrow$ & 0.1716 \\
\hline \multirow[t]{3}{*}{$(0.25,0.25)$} & LMPC & 0.08662 & 0.13446 & 0.16061 & 0.16161 & $\rightarrow$ & 0.16162 \\
\hline & LBIE & 0.08717 & 0.13144 & 0.15728 & 0.15832 & $\rightarrow$ & 0.16162 \\
\hline & MLPG & 0.0902 & 0.1361 & 0.1610 & N/A & $\rightarrow$ & 0.1616 \\
\hline \multirow[t]{3}{*}{$(0.25,0.50)$} & LMPC & 0.07969 & 0.11764 & 0.13772 & 0.13849 & $\rightarrow$ & 0.13850 \\
\hline & LBIE & 0.07991 & 0.11449 & 0.13478 & 0.13558 & $\rightarrow$ & 0.13850 \\
\hline & MLPG & 0.0813 & 0.1223 & 0.1489 & N/A & $\rightarrow$ & 0.1385 \\
\hline \multirow[t]{3}{*}{$(0.00,0.25)$} & LMPC & 0.08904 & 0.13871 & 0.16383 & 0.16474 & $\rightarrow$ & 0.16475 \\
\hline & LBIE & 0.09200 & 0.13915 & 0.16331 & 0.16420 & $\rightarrow$ & 0.16475 \\
\hline & MLPG & 0.0928 & 0.1403 & 0.1641 & N/A & $\rightarrow$ & 0.1648 \\
\hline \multirow[t]{3}{*}{$(0.25,0.00)$} & LMPC & 0.08800 & 0.13902 & 0.16728 & 0.16837 & $\rightarrow$ & 0.16837 \\
\hline & LBIE & 0.08868 & 0.13594 & 0.16386 & 0.16500 & $\rightarrow$ & 0.16837 \\
\hline & MLPG & 0.0917 & 0.1406 & 0.1676 & N/A & $\rightarrow$ & 0.1684 \\
\hline \multirow[t]{3}{*}{$(0.00,0.50)$} & LMPC & 0.08179 & 0.12120 & 0.14049 & 0.14120 & $\rightarrow$ & 0.14121 \\
\hline & LBIE & 0.08411 & 0.12142 & 0.13998 & 0.14066 & $\rightarrow$ & 0.14121 \\
\hline & MLPG & 0.0850 & 0.1225 & 0.1408 & N/A & $\rightarrow$ & 0.1412 \\
\hline \multirow[t]{3}{*}{$(0.25,0.75)$} & LMPC & 0.05733 & 0.07860 & 0.08951 & 0.08993 & $\rightarrow$ & 0.08994 \\
\hline & LBIE & 0.05729 & 0.07687 & 0.08765 & 0.08808 & $\rightarrow$ & 0.08994 \\
\hline & MLPG & 0.0592 & 0.0794 & 0.0898 & N/A & $\rightarrow$ & 0.0899 \\
\hline
\end{tabular}

velocity, along the $x$-axis, for $M=2$ and $\lambda=10,100$ at different time levels, while Fig. 7 shows the velocity, along the $x$-axis, for $\lambda=100$ and $M=4,5$, respectively. In this way, it is possible the comparison of the present results with corresponding results of Figs. 2 and 3, obtained with MLPG method in [28]. Simultaneously, we can see how the Hartmann number and the conductivity parameter $\lambda$, affect the transition time to the steady state.

Figure 8 presents the contour plots of the induced magnetic field for two limiting cases, that is, when the walls are conducting $(\lambda=0)$ and when the walls are insulating $(\lambda \rightarrow \infty)$ at steady state for $M=5$. One can observe that in the conducting walls case $(\lambda=0)$, the induced magnetic field contours are practically perpendicular to the walls. When $\lambda$ increases ( $\lambda \rightarrow \infty$ means the walls are insulated), the induced magnetic field contours obtain the behavior of the solution of the MHD flow with insulated walls. Additionally, Fig. 9 presents the velocity field for different values of wall conductivity.

In order to observe the effect of the Hartmann number $M$ on the flow we present the velocity and the induced magnetic field line contours at Fig. 10, for $M=20,300$ and conductivity parameter $\lambda=0$. In the velocity contour lines, we observe that the flow is separated symmetrically in the $y$-direction, due to the applied magnetic field in the direction of $x$-axis and to the pure conductivity of the wall $(\lambda=0)$. As $M$ increases the separation is more pronounced, the fluid is nearly stagnant at the centre region, while boundary layers are formed close to the boundaries at $y= \pm 1$.

At Fig. 11, a similar behavior for increasing $M$ is depicted for the velocity and the induced magnetic field at $\lambda=5$. It can be noticed that, when the conductivity of the walls in increased, then the fluid is more stagnant at the centre region of the duct.

Finally, in order to demonstrate the applicability of the proposed scheme, we present the contour plots of the induced magnetic field and the velocity field for Hartmann number $M=300$ and high values of conductivity parameter $\lambda=$ 100, 300 at Fig. 12.

\subsection{Unsteady MHD flow in a rectangular duct with insulating walls, under the influence of an oblique magnetic field}

As a second numerical example, we examined a laminar, incompressible and electrically conducting fluid in a square duct with insulating walls where the applied magnetic field configure an angle $\varphi$ with the $y$-axis as shown in Fig. 13 . 
Table 2 Numerical solution of velocity for some selected points at different times for non-conducting walls and $M=20$

\begin{tabular}{|c|c|c|c|c|c|c|c|}
\hline$(\mathrm{x}, \mathrm{y})$ & Numerical method & $t=0.025$ & $t=0.05$ & $t=0.10$ & $t=0.15$ & & Steady state (=exact) \\
\hline \multirow[t]{3}{*}{$(0.00,0.00)$} & LMPC & 0.02489 & 0.04389 & 0.04986 & 0.04991 & $\rightarrow$ & 0.04992 \\
\hline & LBIE & 0.02479 & 0.04345 & 0.04986 & 0.04990 & $\rightarrow$ & 0.04992 \\
\hline & MLPG & N/A & 0.0440 & 0.0499 & 0.0499 & $\rightarrow$ & 0.0499 \\
\hline \multirow[t]{3}{*}{$(0.50,0.00)$} & LMPC & 0.02292 & 0.03712 & 0.04915 & 0.04975 & $\rightarrow$ & 0.04976 \\
\hline & LBIE & 0.02147 & 0.03479 & 0.04877 & 0.04966 & $\rightarrow$ & 0.04976 \\
\hline & MLPG & N/A & 0.0370 & 0.0493 & 0.0498 & $\rightarrow$ & 0.0498 \\
\hline \multirow[t]{3}{*}{$(0.00,0.25)$} & LMPC & 0.02488 & 0.04379 & 0.04960 & 0.04966 & $\rightarrow$ & 0.04966 \\
\hline & LBIE & 0.02478 & 0.04331 & 0.04959 & 0.04962 & $\rightarrow$ & 0.04966 \\
\hline & MLPG & N/A & 0.0439 & 0.0497 & 0.0497 & $\rightarrow$ & 0.0497 \\
\hline \multirow[t]{3}{*}{$(0.25,0.25)$} & LMPC & 0.02459 & 0.04183 & 0.04941 & 0.04956 & $\rightarrow$ & 0.04957 \\
\hline & LBIE & 0.02407 & 0.04010 & 0.04925 & 0.04946 & $\rightarrow$ & 0.04957 \\
\hline & MLPG & N/A & 0.0419 & 0.0485 & 0.0490 & $\rightarrow$ & 0.0496 \\
\hline \multirow[t]{3}{*}{$(0.50,0.25)$} & LMPC & 0.02291 & 0.03703 & 0.04872 & 0.04929 & $\rightarrow$ & 0.04930 \\
\hline & LBIE & 0.02146 & 0.03449 & 0.04827 & 0.04911 & $\rightarrow$ & 0.04930 \\
\hline & MLPG & N/A & 0.0370 & 0.0489 & 0.0493 & $\rightarrow$ & 0.0493 \\
\hline \multirow[t]{3}{*}{$(0.00,0.50)$} & LMPC & 0.02474 & 0.04259 & 0.04766 & 0.04771 & $\rightarrow$ & 0.04772 \\
\hline & LBIE & 0.02459 & 0.04206 & 0.04754 & 0.04757 & $\rightarrow$ & 0.04772 \\
\hline & MLPG & N/A & 0.0428 & 0.0477 & 0.0478 & $\rightarrow$ & 0.0477 \\
\hline \multirow[t]{3}{*}{$(0.25,0.50)$} & LMPC & 0.02445 & 0.04072 & 0.04732 & 0.04744 & $\rightarrow$ & 0.04745 \\
\hline & LBIE & 0.02390 & 0.03879 & 0.04694 & 0.04711 & $\rightarrow$ & 0.04745 \\
\hline & MLPG & N/A & 0.0409 & 0.0475 & 0.0475 & $\rightarrow$ & 0.0475 \\
\hline \multirow[t]{3}{*}{$(0.50,0.50)$} & LMPC & 0.02280 & 0.03608 & 0.04621 & 0.04667 & $\rightarrow$ & 0.04668 \\
\hline & LBIE & 0.02132 & 0.03374 & 0.04549 & 0.04616 & $\rightarrow$ & 0.04668 \\
\hline & MLPG & N/A & 0.0361 & 0.0464 & 0.0468 & $\rightarrow$ & 0.0467 \\
\hline \multirow[t]{3}{*}{$(0.25,0.75)$} & LMPC & 0.02182 & 0.03314 & 0.03721 & 0.03728 & $\rightarrow$ & 0.03730 \\
\hline & LBIE & 0.02125 & 0.03177 & 0.03670 & 0.03679 & $\rightarrow$ & 0.03730 \\
\hline & MLPG & N/A & N/A & N/A & N/A & & \\
\hline
\end{tabular}

Table 3 Numerical solution of velocity for some selected points at different times for non-conducting walls and $M=500$

\begin{tabular}{|c|c|c|c|c|c|c|c|}
\hline$(\mathrm{x}, \mathrm{y})$ & $t=0.015$ & $t=0.025$ & $t=0.040$ & $t=0.050$ & & Steady state (LMPCM) & FEM \\
\hline$(0.00,0.00)$ & 0.001913 & 0.001750 & 0.001981 & 0.002080 & $\rightarrow$ & 0.002000 & 0.002000 \\
\hline$(0.50,0.00)$ & 0.001995 & 0.001959 & 0.001967 & 0.002008 & $\rightarrow$ & 0.002000 & 0.002000 \\
\hline$(0.00,0.25)$ & 0.001913 & 0.001750 & 0.001981 & 0.002080 & $\rightarrow$ & 0.002000 & 0.002000 \\
\hline$(0.25,0.25)$ & 0.001932 & 0.001808 & 0.001984 & 0.002051 & $\rightarrow$ & 0.002000 & 0.002000 \\
\hline$(0.50,0.25)$ & 0.001995 & 0.001959 & 0.001967 & 0.002008 & $\rightarrow$ & 0.002000 & 0.002000 \\
\hline$(0.00,0.50)$ & 0.001913 & 0.001750 & 0.001981 & 0.002080 & $\rightarrow$ & 0.002000 & 0.002000 \\
\hline$(0.25,0.50)$ & 0.001932 & 0.001808 & 0.001984 & 0.002051 & $\rightarrow$ & 0.002000 & 0.002000 \\
\hline$(0.50,0.50)$ & 0.001995 & 0.001959 & 0.001967 & 0.002008 & $\rightarrow$ & 0.002000 & 0.001999 \\
\hline$(0.25,0.75)$ & 0.001932 & 0.001808 & 0.001984 & 0.002051 & $\rightarrow$ & 0.002000 & 0.002000 \\
\hline
\end{tabular}

The numerical solutions of various Hartmann numbers are obtained for several time steps and at selected nodes. The results for $M=5, M=50$ and $M=500$ and angle $\varphi=\frac{\pi}{3}$ are presented. The number of nodes used is 1681 , 1681 and 6561 respectively, and $\delta t=0.005$, the same as before, since low and moderate Hartmann numbers are examined. In Tables 4 and 5, the values of the velocity $V$ are presented for future validation. Contour plots of the velocity field and the induced magnetic field for Hartmann numbers $M=50,100,500$ are presented at Fig. 14 . 


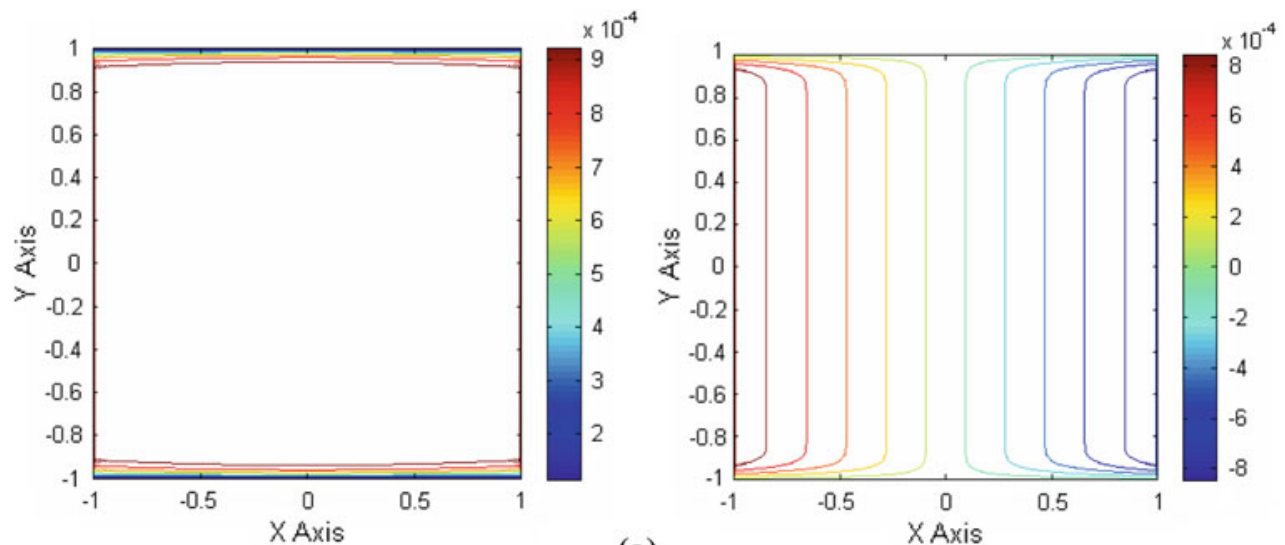

(a)
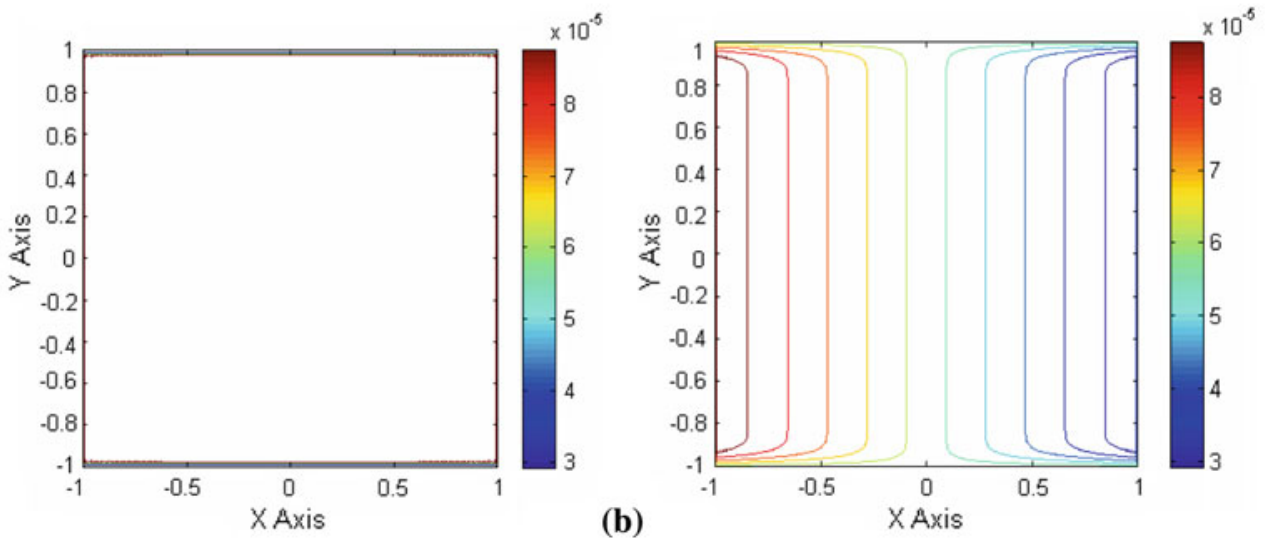

Fig. 5 Contours of velocity and induced magnetic field for $\mathbf{a} M=1,000$ and $\mathbf{b} M=10,000$ for $\lambda \rightarrow \infty$
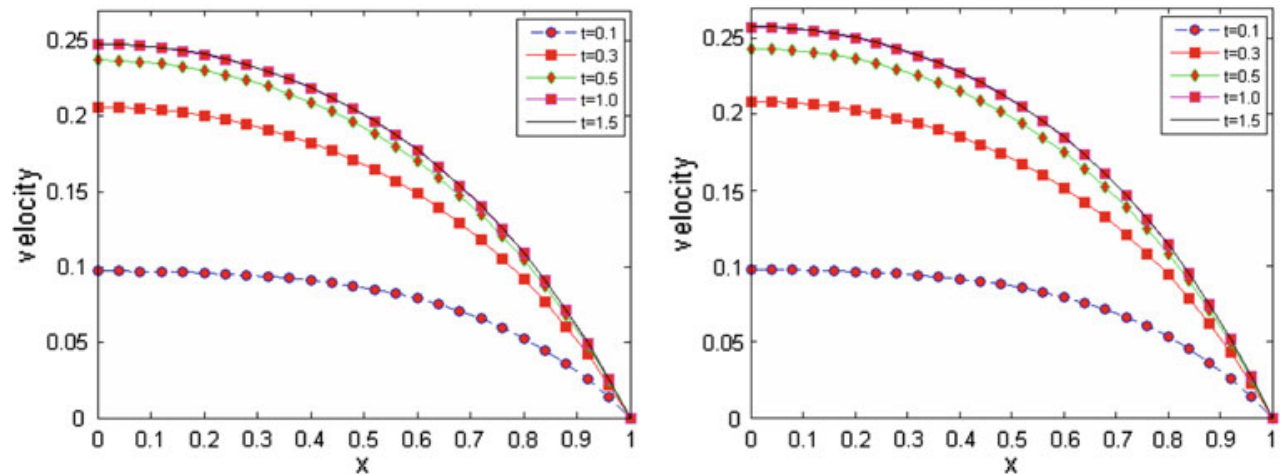

Fig. 6 Velocity along the $x$-axis for $M=2$ and $\lambda=10,100$ at different time levels

\subsection{Unsteady MHD flow in a triangular duct with insulating walls}

As an irregular cross section for an MHD flow, we examined the case of a triangular cross section as shown in Fig. 15. The applied magnetic field is oblique, having an angle $\varphi$ with the $y$-axis.

The geometry in Fig. 15 is irregular and, inevitably, it is difficult to have the nodes regularly distributed. However, this obstacle has been overcame, following a procedure devel- oped in [37]. Therein, a nodal distribution of the Type-I was used, embedded at the prescribed geometry and, ensuring the convergence and the stability of the discrete harmonic operator [38]. Defining the methodology for the construction of an embedded regular grid of Type-I, we address the following steps. Initially, the spatial dimensions of the geometry are defined. Then, a regular grid containing the geometry is constructed (Fig. 16). Finally, the grid is confined into the boundaries of the geometry. Attention should be taken, such that no degenerated nodes on the boundary exist. 

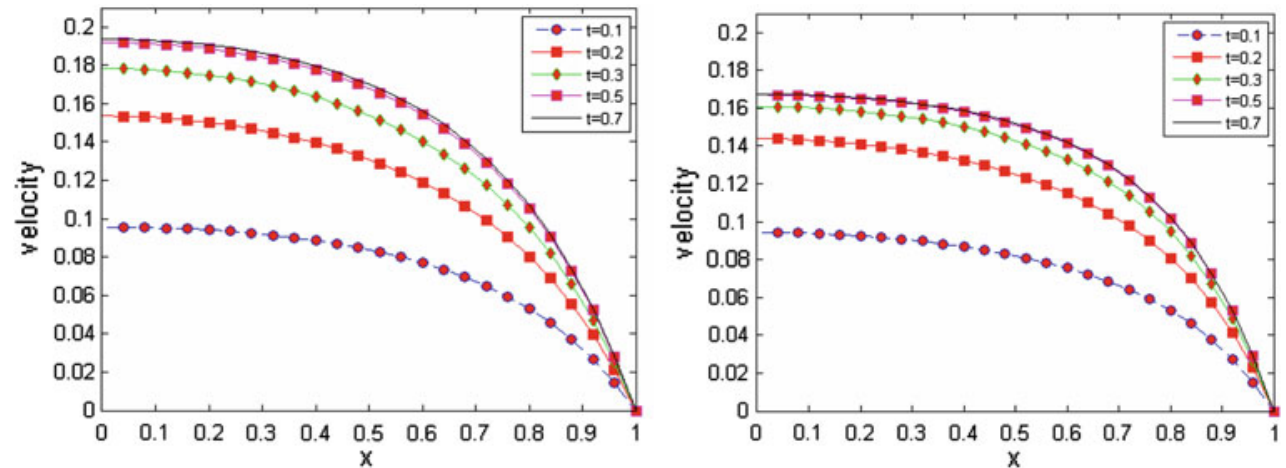

Fig. 7 Velocity along the $x$-axis for $\lambda=100$ and $M=4,5$ at different time levels

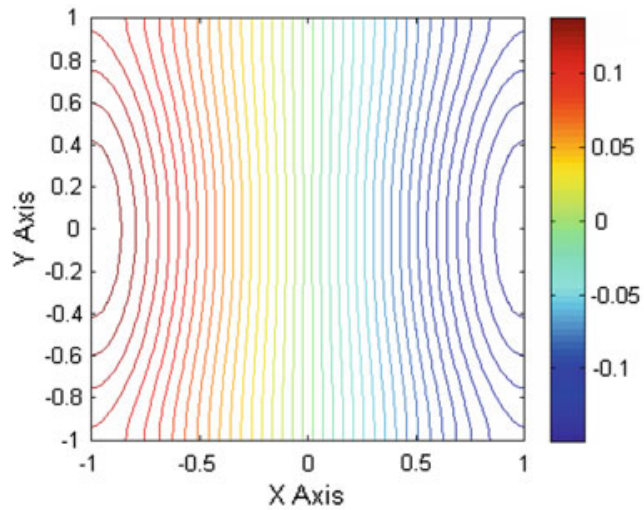

(a)

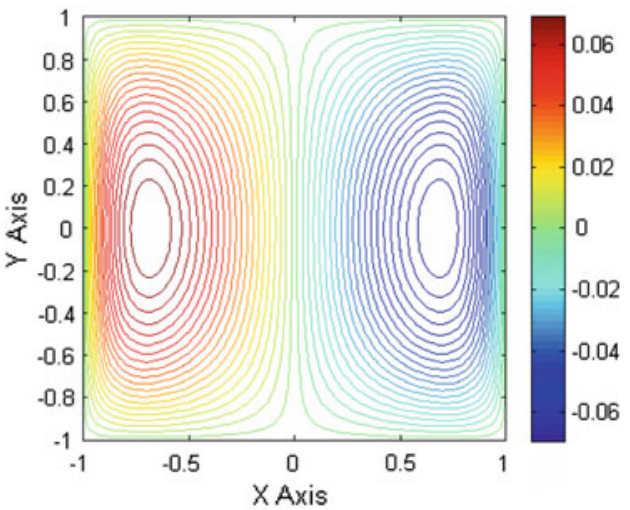

(b)

Fig. 8 Induced magnetic field for a variable conductivity with $\lambda=0$; $\mathbf{b}$ insulated walls for $(\lambda \rightarrow \infty), M=5$

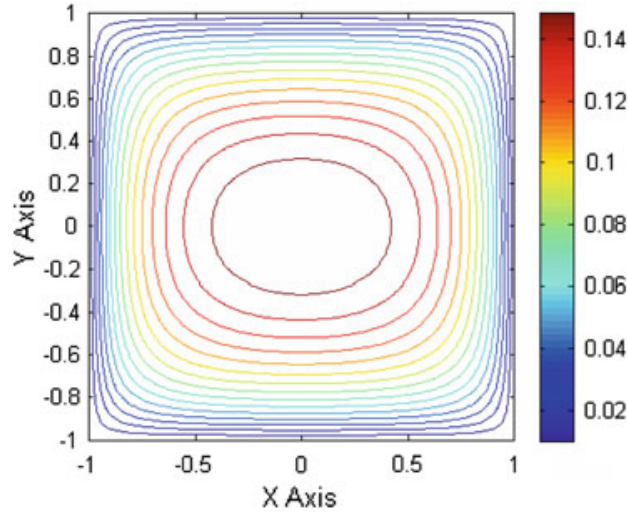

(a)

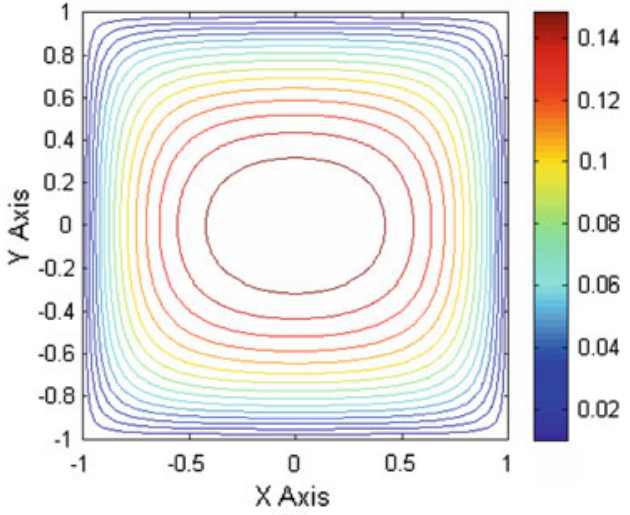

(b)

Fig. 9 Velocity field for variable conductivity with $\mathbf{a} \lambda=5 ; \mathbf{b} \lambda=30$

For the triangular pipe with non-conducting walls $(\lambda \rightarrow \infty)$, the steady state numerical solution is also obtained. Hartmann numbers up to $M=100,000$ were examined and the results for the velocity and the induced magnetic field are presented at prescribed locations. The numerical solutions of the velocity field and the induced magnetic field are presented at Table 6 for Hartmann number $M=500$. The values are calculated at selected points for distinct time 
Fig. 10 Contours of velocity and induced magnetic field for $\lambda=0$ and $\mathbf{a} M=20$,

b $M=300$

Fig. 11 Contours of velocity and induced magnetic field for $\lambda=5$ and $\mathbf{a} M=50$,

b $M=300$
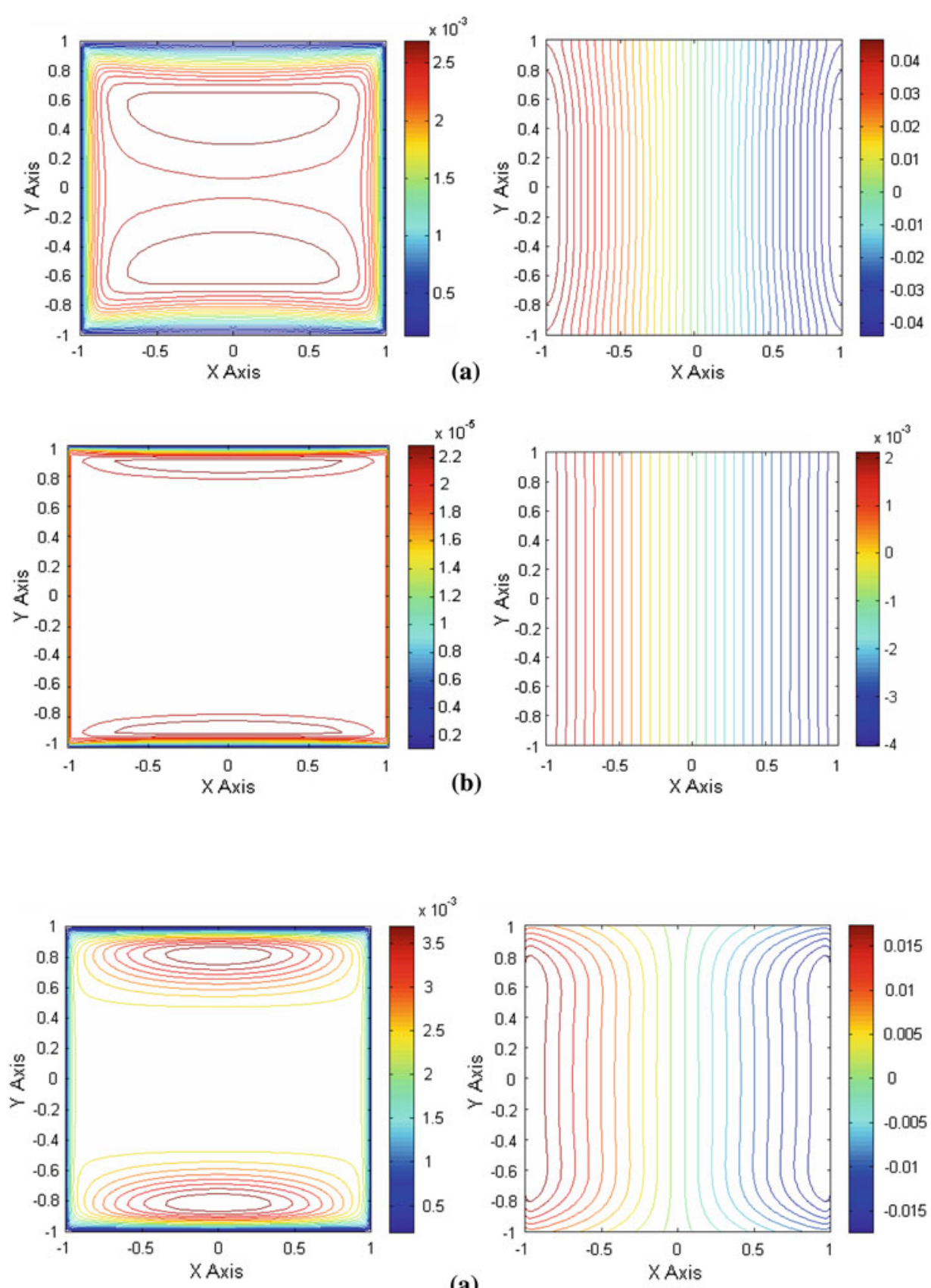

(a)
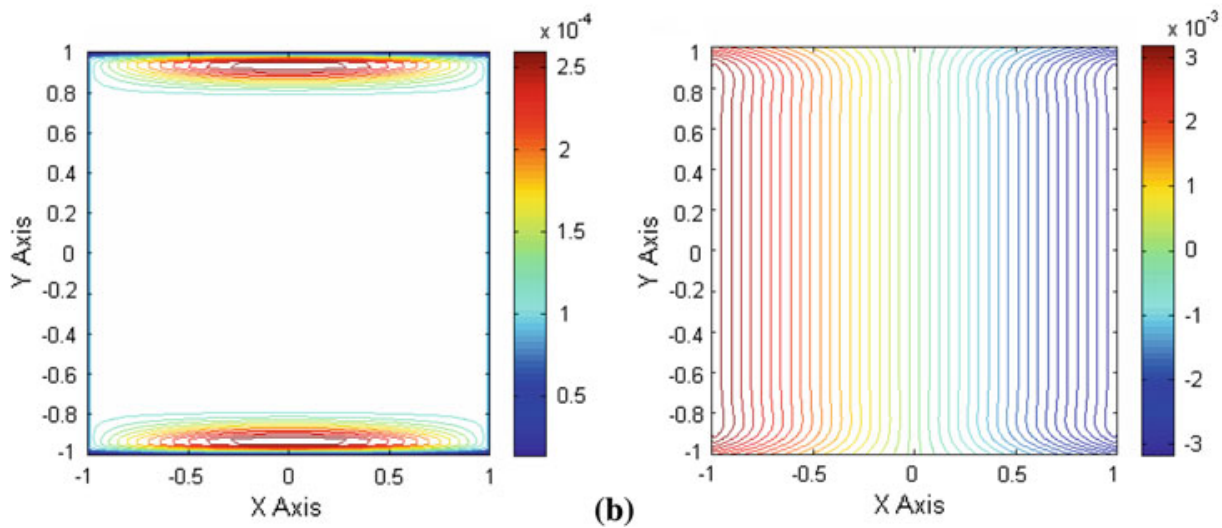

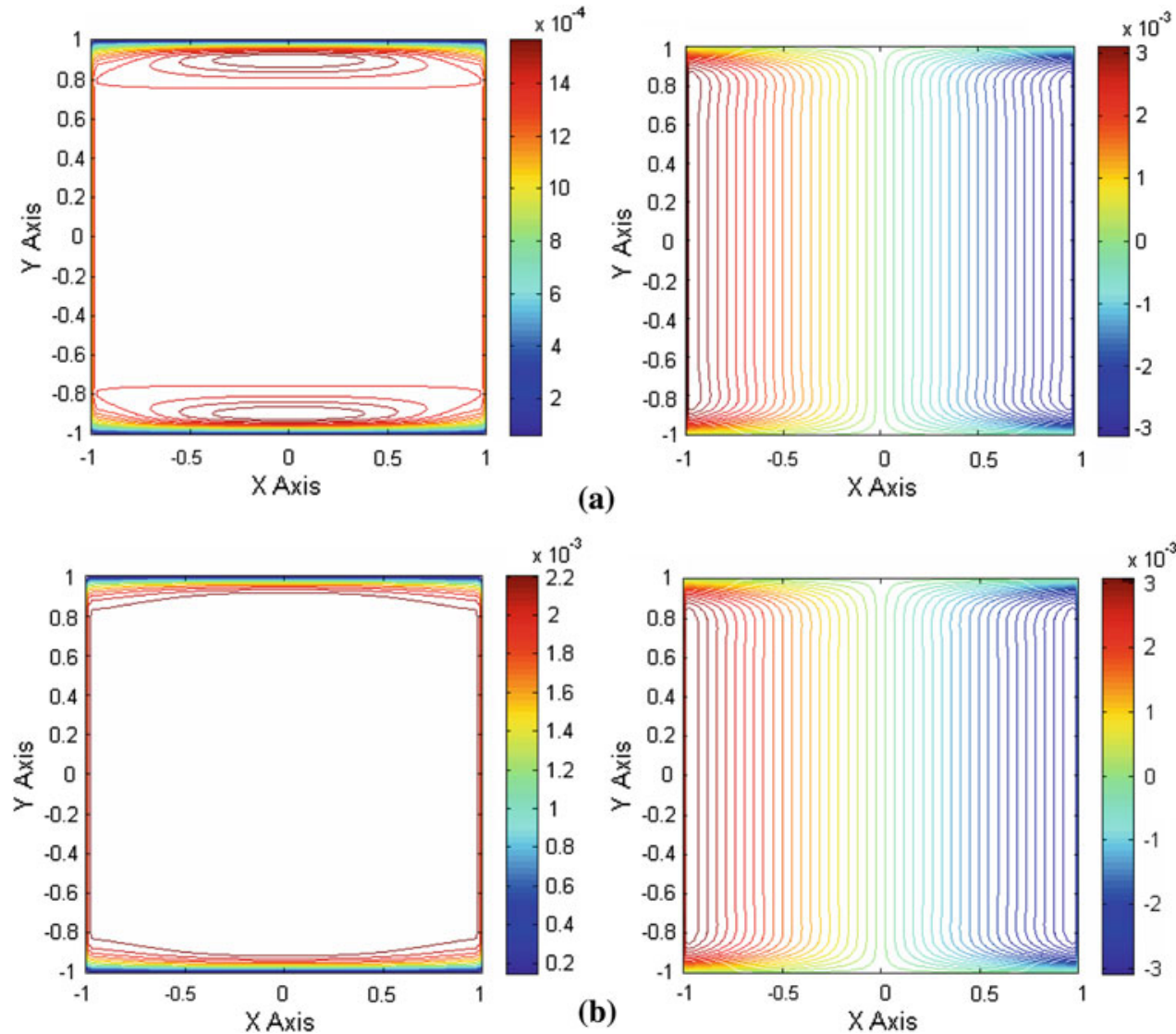

Fig. 12 Contours of velocity and induced magnetic field for $M=300$ when $\mathbf{a} \lambda=100$ and $\mathbf{b} \lambda=300$

steps. As the Hartmann number gradually increases, the time step $\delta t$ needs to obtain smaller values in order to increase the accuracy. Thus, for moderate Hartmann numbers $(100 \leq$ $M \leq 1,000)$ the calculations are performed using a time step $\delta t=0.001$ and for total number $N=4,961$ of regularly distributed nodes. Boundary layers are formed as the Hartmann number increases, and the numerical solutions are very sensitive to the presence of unwanted oscillations.

The unwanted oscillations alter the numerical results, giving inaccurate solutions. Several strategies for meshless methods were developed, such as nodal refinement, enlargement of the local support domain, fully upwind support domain, and adaptive upwind support domain [39]. All the aforementioned methods have several advantages and disadvantages. In view of nodal refinement, the increase at the number of nodes offers more accurate results, although there is a proportional increase in the computational time. By enlarging the local support domain one captures the upstream information more efficiently but the accuracy of the solution is reduced [27]. This can be more evident whenever regions with high gradients are present. By using an upwind support domain, the accuracy and stability is improved for problems

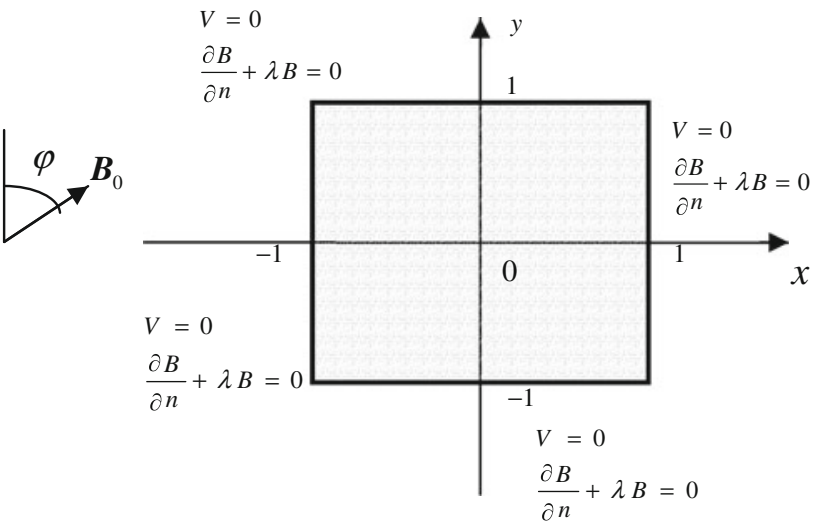

Fig. 13 Square section of the duct with variable wall conductivity

with high Hartmann numbers, still it gives very poor results for low and moderate Hartmann ones. Thus, the nodal refinement with an aspect ratio 2:1 was adopted. In Fig. 17 the plots of the velocity and the induced magnetic field are presented for Hartmann numbers $M=100,1,000$ and 10,000, without the presence of the unwanted oscillations and following 
Table 4 Numerical solution of velocity for some selected points at different times for non-conducting walls and $M=5,50$

\begin{tabular}{|c|c|c|c|c|c|c|}
\hline$(\mathrm{x}, \mathrm{y})$ & $t=0.1$ & $t=0.2$ & $t=0.5$ & $t=1.0$ & & Steady state $(=$ exact $)$ \\
\hline \multicolumn{7}{|l|}{$(0.00,0.00)$} \\
\hline$M=5$ & 0.09086 & 0.14546 & 0.17431 & 0.17542 & $\rightarrow$ & 0.17543 \\
\hline$M=50$ & 0.02287 & 0.02287 & 0.02287 & 0.02287 & $\rightarrow$ & 0.02288 \\
\hline \multicolumn{7}{|l|}{$(0.25,0.25)$} \\
\hline$M=5$ & 0.08727 & 0.13828 & 0.16880 & 0.17000 & $\rightarrow$ & 0.17001 \\
\hline$M=50$ & 0.02286 & 0.02286 & 0.02286 & 0.02286 & $\rightarrow$ & 0.02286 \\
\hline \multicolumn{7}{|l|}{$(0.25,0.50)$} \\
\hline$M=5$ & 0.08098 & 0.12475 & 0.15281 & 0.15408 & $\rightarrow$ & 0.15409 \\
\hline$M=50$ & 0.02210 & 0.02210 & 0.02210 & 0.02210 & $\rightarrow$ & 0.02210 \\
\hline \multicolumn{7}{|l|}{$(0.00,0.25)$} \\
\hline$M=5$ & 0.08931 & 0.14091 & 0.16847 & 0.16954 & $\rightarrow$ & 0.16955 \\
\hline$M=50$ & 0.02242 & 0.02242 & 0.02242 & 0.02242 & $\rightarrow$ & 0.02242 \\
\hline \multicolumn{7}{|l|}{$(0.25,0.00)$} \\
\hline$M=5$ & 0.08858 & 0.14068 & 0.16983 & 0.17099 & $\rightarrow$ & 0.17100 \\
\hline$M=50$ & 0.02263 & 0.02263 & 0.02263 & 0.02263 & $\rightarrow$ & 0.02263 \\
\hline \multicolumn{7}{|l|}{$(0.00,0.50)$} \\
\hline$M=5$ & 0.08245 & 0.12506 & 0.14847 & 0.14942 & $\rightarrow$ & 0.14943 \\
\hline$M=50$ & 0.02057 & 0.02057 & 0.02057 & 0.02057 & $\rightarrow$ & 0.02057 \\
\hline \multicolumn{7}{|l|}{$(0.25,0.75)$} \\
\hline$M=5$ & 0.06157 & 0.09062 & 0.11044 & 0.11139 & $\rightarrow$ & 0.11140 \\
\hline$M=50$ & 0.01939 & 0.01939 & 0.01939 & 0.01939 & $\rightarrow$ & 0.01939 \\
\hline
\end{tabular}

the nodal refinement. Finally, in Fig. 18, we present results for the case of irregular nodal distribution. In this case, the ensuring of positivity conditions [30] is a prerequisite for the convergence of the numerical solution.

\subsection{Unsteady MHD flow in a circular duct with insulating walls}

As a second example of an irregular cross section MHD flow, we examined the case of a circular cross section as shown at Fig. 19.

The procedure presented before, for the nodal distribution description and embedded grids, is adopted. The applied magnetic field is perpendicular to the $y$-axis while, Hartmann numbers up to $M=100,000$ were examined. We present the numerical solution for the MHD flow on a circular pipe $x^{2}+y^{2}=1$ for Hartmann numbers $M=5,100$ and 200 (Fig. 20).

In Table 7 the numerical results of the velocity and the magnetic fields are presented at selected points and at different time steps for Hartmann number $M=5$. The grid used here was a regular, Type-I, $41 \times 41$ grid and the total number of nodes used was 1,681 (where the 180 nodes was along the circumference of the circular disk). The numerical results of the meshless point collocation method are in a good agreement with the corresponding ones obtained using the mesh-
Table 5 Numerical solution of velocity for some selected points at different times for non-conducting walls at $M=500$

\begin{tabular}{llllll}
\hline$(\mathrm{x}, \mathrm{y})$ & $t=0.025$ & $t=0.05$ & $t=0.5$ & & Steady state \\
\hline$(0.00,0.00)$ & $0.96332 \mathrm{e}-03$ & $0.00111 \mathrm{e}-03$ & $0.00101 \rightarrow 0.00230$ \\
$(0.25,0.25)$ & $0.96627 \mathrm{e}-03$ & $0.00108 \mathrm{e}-03$ & $0.00098 \rightarrow 0.00230$ \\
$(0.25,0.50)$ & $0.96627 \mathrm{e}-03$ & $0.00108 \mathrm{e}-03$ & $0.00098 \rightarrow 0.00230$ \\
$(0.00,0.25)$ & $0.96332 \mathrm{e}-03$ & $0.00111 \mathrm{e}-03$ & $0.00101 \rightarrow 0.00230$ \\
$(0.25,0.00)$ & $0.96627 \mathrm{e}-03$ & $0.00108 \mathrm{e}-03$ & $0.00098 \rightarrow 0.00230$ \\
$(0.00,0.50)$ & $0.96332 \mathrm{e}-03$ & $0.00111 \mathrm{e}-03$ & $0.00101 \rightarrow 0$ & $\rightarrow 0.0214$ \\
$(0.25,0.75)$ & $0.96627 \mathrm{e}-03$ & $0.00108 \mathrm{e}-03$ & $0.00098 \rightarrow 0.00194$
\end{tabular}

less local boundary integral method (LBIE) [27], as well as, with results obtained with FEM and BEM method [40].

As aforementioned, when the Hartmann number increases, then boundary layers are formed and the numerical solutions become sensitive to the presence of unwanted oscillations. In order to attain accuracy, the total number of nodes is increased and the time step $\delta t$ is decreased to smaller values. For moderate Hartmann numbers the calculations were performed using time step $\delta t=0.001$. At Table 8 numerical results are presented at prescribed points and various time steps for Hartmann number $M=100$. 

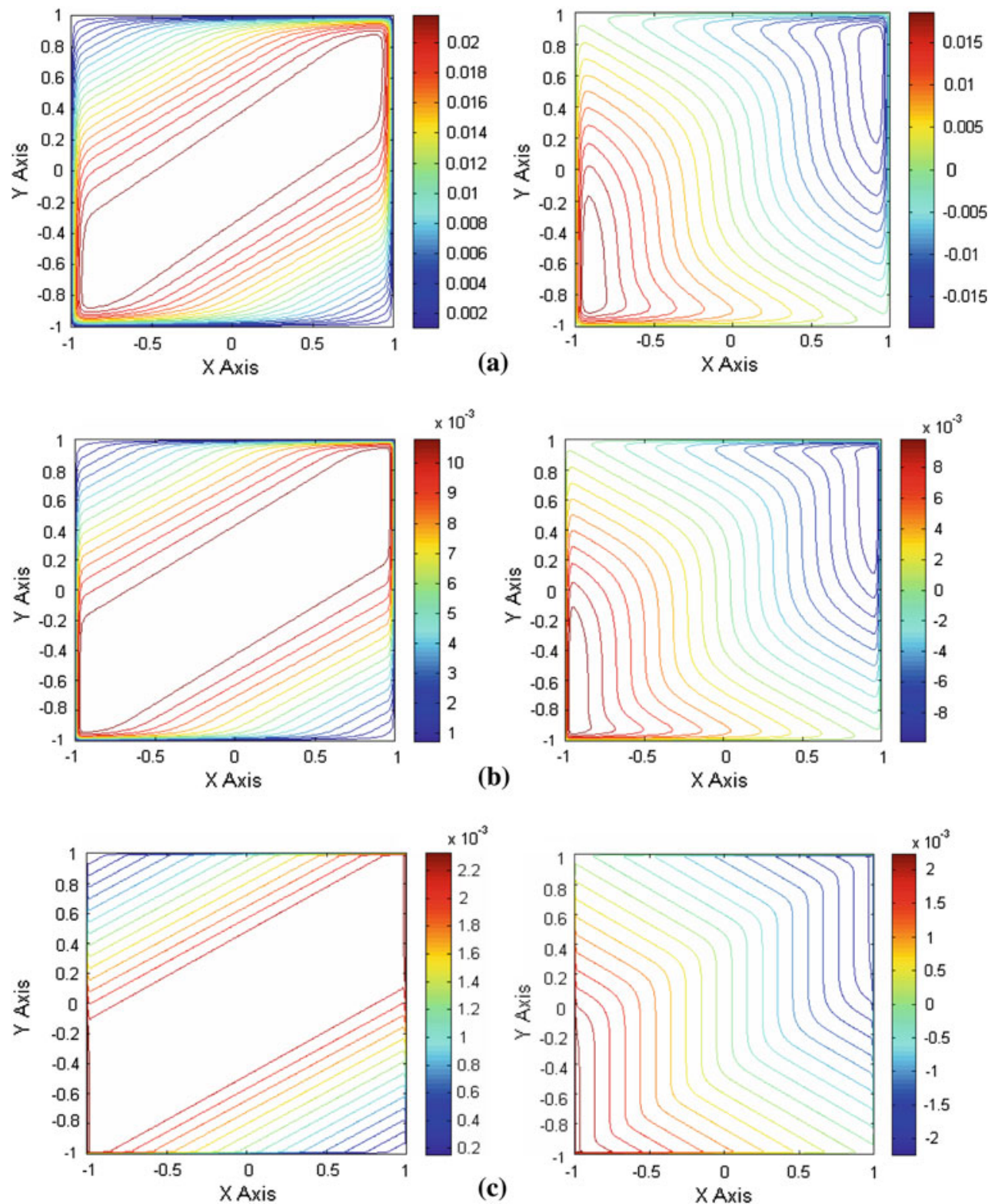

Fig. 14 Contours of velocity and induced magnetic field for $\varphi=\frac{\pi}{3}$ and $\lambda \rightarrow \infty$, when a $M=50$, b $M=100$, c $M=500$

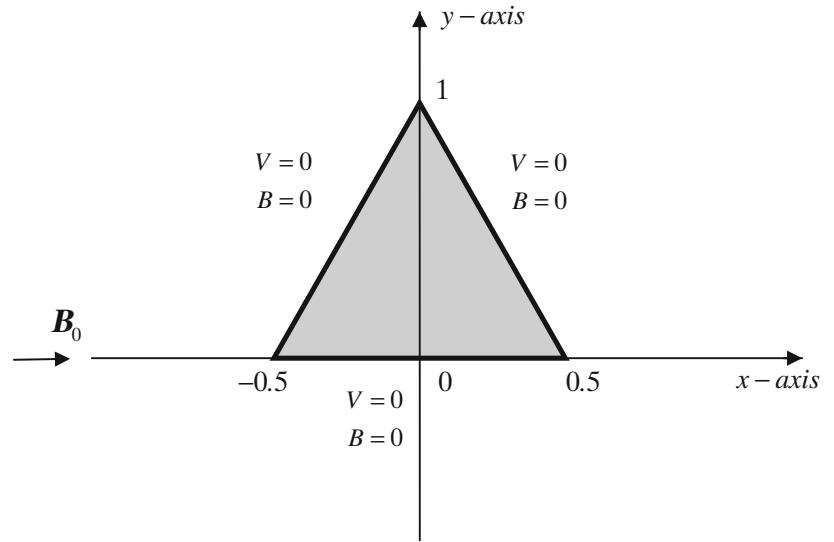

Fig. 15 Triangular section of the duct with variable wall conductivity

\section{Convergence and efficiency of the proposed scheme}

In order to examine the accuracy and the efficiency of the proposed scheme, results showing the errors norms $L_{2}$ and $L_{\infty}$, the CPU time for different grid sizes, as well as the convergence rate, are presented.

The accuracy of the scheme is measured computing the following error norms, that is, the $L_{2}$ and $L_{\infty}$ defined as

$$
L_{2}=\left\|u_{\text {exact }}-u_{\text {num }}\right\|_{2}=\sqrt{h \sum_{j=0}^{N}\left|\left(u_{\text {exact }}\right)_{j}-\left(u_{\text {num }}\right)_{j}\right|^{2}}
$$


Fig. 16 a Regular grid containing the geometry, b Grid conformed at the geometry

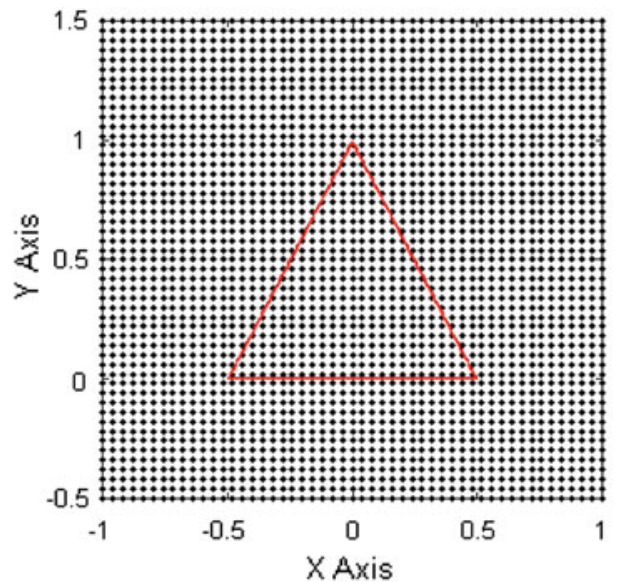

(a)

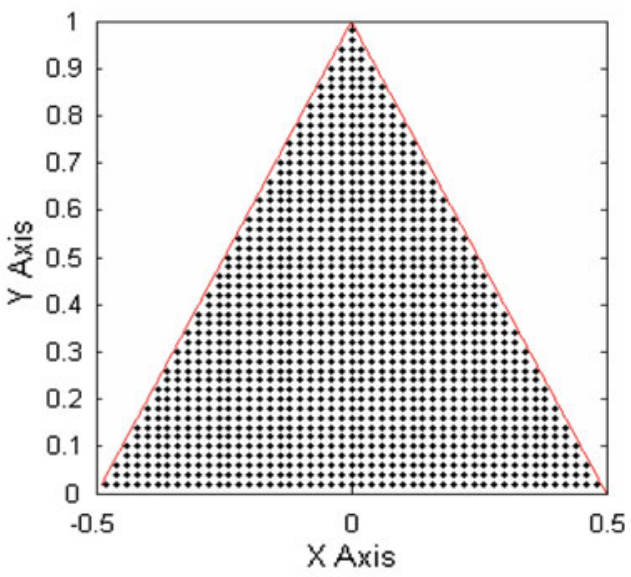

(b)

Table 6 Numerical solution of velocity and induced magnetic field for some selected points at different times for non-conducting walls and $M=500$

\begin{tabular}{lrrrrrr}
\hline $\mathrm{x}, \mathrm{y})$ & $t=0.1$ & $t=0.3$ & $t=0.5$ & $t=1.0$ & & Steady state \\
\hline $\begin{array}{l}(0.10,0.30) \\
u\end{array}$ & $0.7098 \mathrm{e}-003$ & $0.6984 \mathrm{e}-003$ & $0.7069 \mathrm{e}-003$ & $0.7045 \mathrm{e}-003$ & $\rightarrow$ & $0.7000 \mathrm{e}-003$ \\
$B$ & $-0.1558 \mathrm{e}-003$ & $-0.2060 \mathrm{e}-003$ & $-0.1994 \mathrm{e}-003$ & $-0.2004 \mathrm{e}-003$ & $\rightarrow$ & $-0.2000 \mathrm{e}-003$ \\
$(0.10,0.60)$ & & & & & & \\
$u$ & $0.4156 \mathrm{e}-003$ & $0.4116 \mathrm{e}-003$ & $0.3933 \mathrm{e}-003$ & $0.4009 \mathrm{e}-003$ & $\rightarrow$ & $0.3999 \mathrm{e}-003$ \\
$B$ & $0.2264 \mathrm{e}-003$ & $-0.1996 \mathrm{e}-003$ & $-0.2004 \mathrm{e}-003$ & $-0.1993 \mathrm{e}-003$ & $\rightarrow$ & $-0.1999 \mathrm{e}-003$ \\
$(0.30,0.30)$ & & & & & & \\
$u$ & $0.6527 \mathrm{e}-003$ & $0.6973 \mathrm{e}-003$ & $0.7005 \mathrm{e}-003$ & $0.7011 \mathrm{e}-003$ & $\rightarrow$ & $0.7016 \mathrm{e}-003$ \\
$B$ & $-0.5885 \mathrm{e}-003$ & $-0.5910 \mathrm{e}-003$ & $-0.5941 \mathrm{e}-003$ & $-0.6019 \mathrm{e}-003$ & $\rightarrow$ & $-0.6016 \mathrm{e}-003$ \\
$(0.30,0.40)$ & & & & & & \\
$u$ & $-0.0000 \mathrm{e}-003$ & $-0.0000 \mathrm{e}-003$ & $-0.0000 \mathrm{e}-003$ & $-0.0000 \mathrm{e}-003$ & $\rightarrow$ & $0.0000 \mathrm{e}-003$ \\
$B$ & $0.0000 \mathrm{e}-003$ & $0.0000 \mathrm{e}-003$ & $0.0000 \mathrm{e}-003$ & $0.0000 \mathrm{e}-003$ & $\rightarrow$ & $-0.0000 \mathrm{e}-003$ \\
\hline
\end{tabular}

and

$L_{\infty}=\left\|u_{\text {exact }}-u_{\text {num }}\right\|_{\infty}=\max _{j}\left|\left(u_{\text {exact }}\right)_{j}-\left(u_{\text {num }}\right)_{j}\right|$

where $u_{\text {exact }}$ and $u_{\text {num }}$ represent the exact and approximate solutions respectively and $h$ is the minimum distance between any two collocation points of the domain set, since the node distribution is uniform. The corresponding results are presented in Table 9. Considering the run time of the meshless methods, the shape functions are not pre-defined and they must be constructed once, before the numerical solution of the resulting algebraic system. Thus, in our in-house code, the numerical procedure is primarily decomposed into two parts. Initially, the construction of the shape functions takes place, and then the solution of the resulting linear system is addressed. The CPU time (in seconds) for the prescribed number of nodes is shown only for the solution of the resulting algebraic linear system. The hardware characteristics used for this benchmarking are trivial, such as a CPU Pentium IV, $2.4 \mathrm{~Hz}$ with $2 \mathrm{~GB}$ RAM. From the numerical results given in Table 9, it is clear that the performance of the proposed meshfree method is both accurate and efficient.

Since we have computed the $L_{\infty}$ norm for each $h_{i}$ we are able to compute the convergence rate of the proposed scheme. For this reason in Fig. 21a we plot the $-\log _{10}\left(L_{\infty}\right)$ as the vertical axis against $\log _{10}\left(h_{i}\right)$ taken as the horizontal axis and, the slope of the plotted line is the convergence rate. Corresponding results are presented in Fig. 21b for the convergence rate when $\delta t$ is decreased. Computations are carried out with different spatial and time step sizes to examine the point rates of convergence in space and in time. 

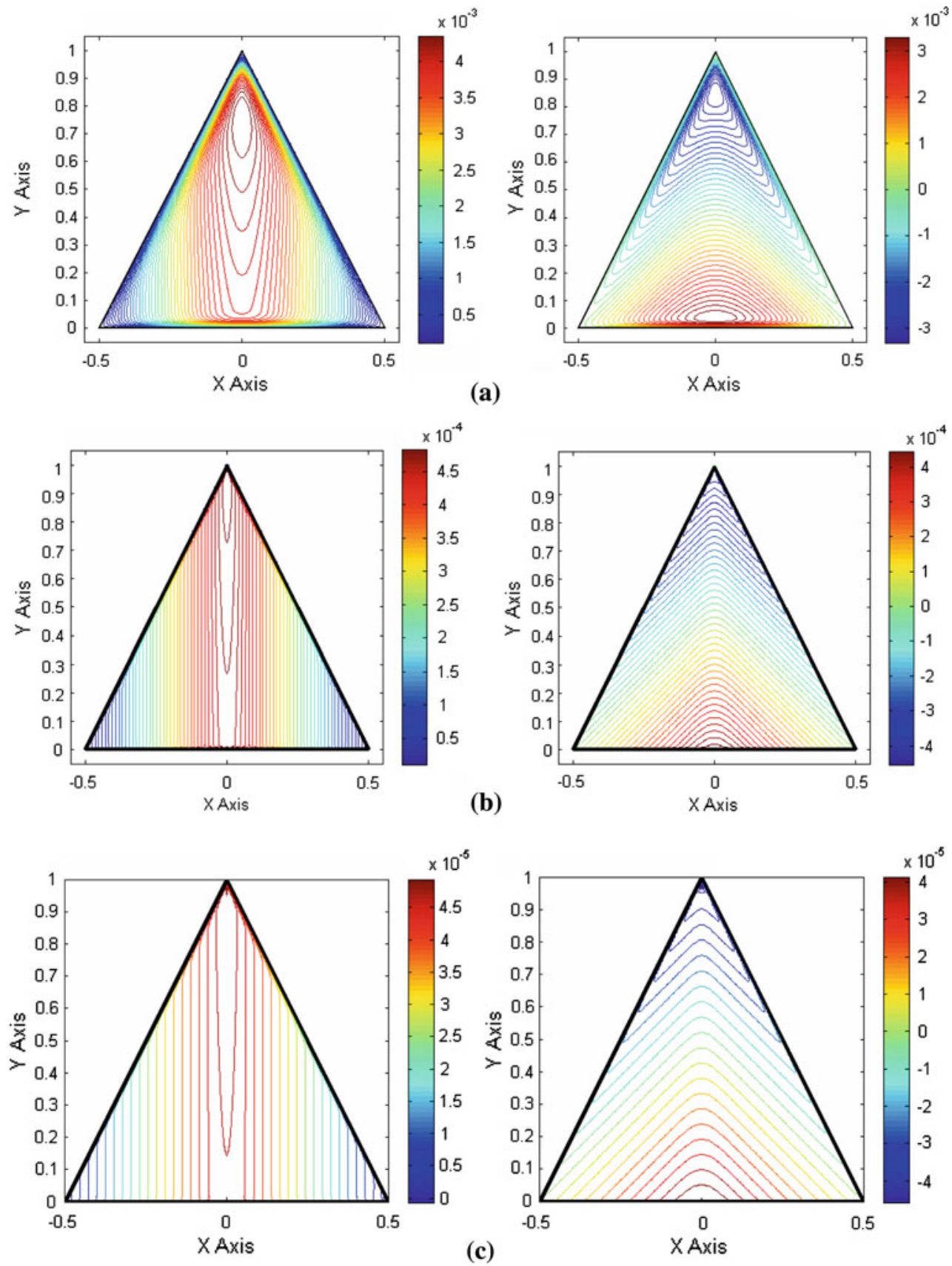

Fig. 17 Contour plots of the velocity field and the induced magnetic field for a $M=100, \mathbf{b} M=1,000$ and $\mathbf{c} M=10,000$

Regarding of the convergence rate in time for the proposed scheme, numerical tests were performed with different time steps $\delta t=0.1,0.05,0.025,0.005$ for $M=5$ and $M=20$. More precisely, the number of the collocation points is kept fixed at $N=1681(41 \times 41)$ and the time step size varies in order to compute the time rate of convergence. The numerical results are computed at time $t=1$, where the transient problem reaches the steady state. The numerical results are listed in Table 10.

\section{Conclusions}

The strong-form localized meshless point collocation (LMPC) formulation with "interpolating" MLS shape functions were developed for the numerical solution of the time dependent, coupled system equations of magnetohydrodynamics flow for regular and irregular domains. Regular and irregular nodal distributions were used. Arbitrary wall conductivity conditions were applied and a uniform magnetic 
Fig. 18 Irregular nodal distribution and contour plots of the induced magnetic field for $M=1,000$
Table 7 Numerical solution of velocity and magnetic field for some selected points at different time levels for $M=5$
For every value of $t$, the first and second rows of data correspond to the use of LMPC and LBIE method, respectively. Moreover, the third and fourth rows of data correspond to results obtained using FEM and BEM method, respectively
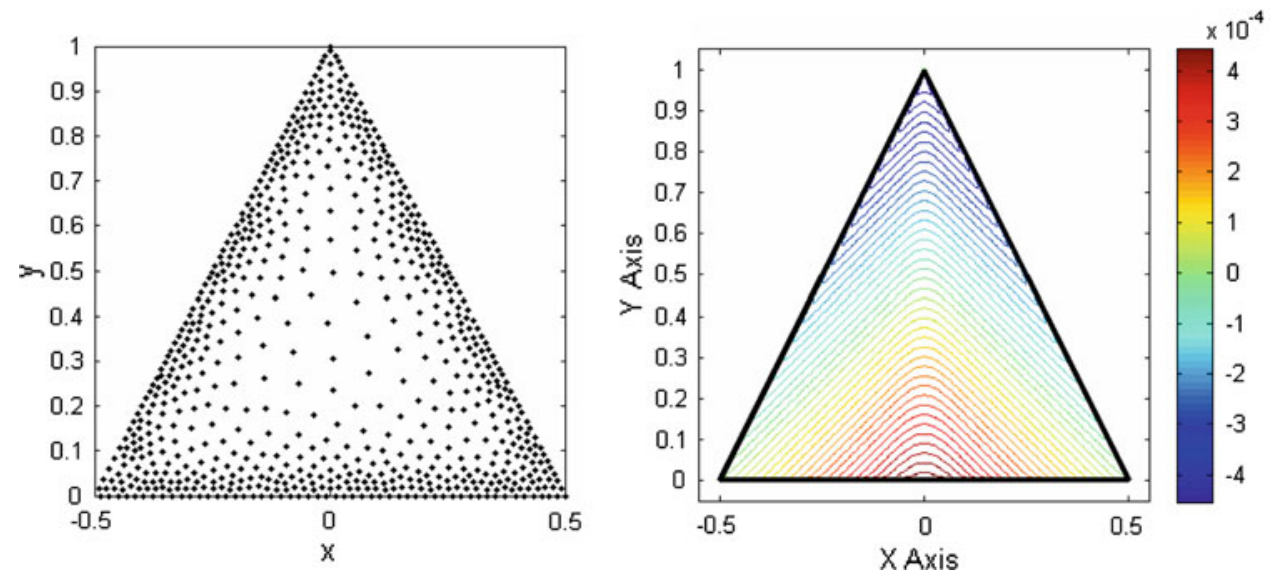

\begin{tabular}{|c|c|c|c|c|c|c|c|}
\hline$(\mathrm{x}, \mathrm{y})$ & Numerical method & $t=0.1$ & $t=0.2$ & $t=0.5$ & $t=1.0$ & & Steady state \\
\hline \multicolumn{8}{|l|}{$u$} \\
\hline \multirow[t]{4}{*}{$(0.0,0.0)$} & LMPC & 0.0918 & 0.1341 & 0.1525 & 0.1530 & $\rightarrow$ & 0.1530 \\
\hline & LBIE & 0.0913 & 0.1339 & 0.1527 & 0.1530 & $\rightarrow$ & 0.1530 \\
\hline & FEM & N/A & N/A & N/A & N/A & $\rightarrow$ & 0.1530 \\
\hline & BEM & N/A & N/A & N/A & N/A & $\rightarrow$ & 0.1530 \\
\hline \multirow[t]{4}{*}{$(1 / 3,0.0)$} & LMPC & 0.0862 & 0.1263 & 0.1461 & 0.1466 & $\rightarrow$ & 0.1466 \\
\hline & LBIE & 0.0858 & 0.1260 & 0.1462 & 0.1466 & $\rightarrow$ & 0.1466 \\
\hline & FEM & N/A & N/A & N/A & N/A & $\rightarrow$ & 0.1466 \\
\hline & BEM & N/A & N/A & N/A & N/A & $\rightarrow$ & 0.1466 \\
\hline \multirow[t]{4}{*}{$(2 / 3,0.0)$} & LMPC & 0.0658 & 0.0968 & 0.1159 & 0.1165 & $\rightarrow$ & 0.1165 \\
\hline & LBIE & 0.0659 & 0.0970 & 0.1163 & 0.1165 & $\rightarrow$ & 0.1165 \\
\hline & FEM & N/A & N/A & N/A & N/A & $\rightarrow$ & 0.1165 \\
\hline & BEM & N/A & N/A & N/A & N/A & $\rightarrow$ & 0.1165 \\
\hline \multirow[t]{4}{*}{$(0.0,2 / 3)$} & LMPC & 0.0636 & 0.0832 & 0.0916 & 0.0918 & $\rightarrow$ & 0.0918 \\
\hline & LBIE & 0.0635 & 0.0834 & 0.0917 & 0.0918 & $\rightarrow$ & 0.0918 \\
\hline & FEM & N/A & N/A & N/A & N/A & $\rightarrow$ & 0.0918 \\
\hline & BEM & N/A & N/A & N/A & N/A & $\rightarrow$ & 0.0918 \\
\hline \multicolumn{8}{|l|}{$-B$} \\
\hline \multirow[t]{4}{*}{$(0.0,0.0)$} & LMPC & 0.0000 & 0.0000 & 0.0000 & 0.0000 & $\rightarrow$ & 0.0000 \\
\hline & LBIE & 0.0000 & 0.0000 & 0.0000 & 0.0000 & $\rightarrow$ & 0.0000 \\
\hline & FEM & N/A & N/A & N/A & N/A & $\rightarrow$ & 0.0000 \\
\hline & BEM & N/A & N/A & N/A & N/A & $\rightarrow$ & 0.0000 \\
\hline \multirow[t]{4}{*}{$(1 / 3,0.0)$} & LMPC & 0.0096 & 0.0281 & 0.0404 & 0.0408 & $\rightarrow$ & 0.0408 \\
\hline & LBIE & 0.0096 & 0.0278 & 0.0401 & 0.0408 & $\rightarrow$ & 0.0408 \\
\hline & FEM & N/A & N/A & N/A & N/A & $\rightarrow$ & 0.0407 \\
\hline & BEM & N/A & N/A & N/A & N/A & $\rightarrow$ & 0.0407 \\
\hline \multirow[t]{4}{*}{$(2 / 3,0.0)$} & LMPC & 0.0200 & 0.0448 & 0.0619 & 0.0624 & $\rightarrow$ & 0.0624 \\
\hline & LBIE & 0.0201 & 0.0448 & 0.0623 & 0.0624 & $\rightarrow$ & 0.0624 \\
\hline & FEM & N/A & N/A & N/A & N/A & $\rightarrow$ & 0.0624 \\
\hline & BEM & N/A & N/A & N/A & N/A & $\rightarrow$ & 0.0624 \\
\hline
\end{tabular}


Table 8 Numerical solution of velocity and magnetic field for some selected points at different time levels for $M=100$

\begin{tabular}{|c|c|c|c|c|c|c|}
\hline$(\mathrm{x}, \mathrm{y})$ & $t=0.005$ & $t=0.01$ & $t=0.015$ & $t=1.0$ & & Steady state \\
\hline \multicolumn{7}{|l|}{$u$} \\
\hline$(0.0,0.0)$ & 0.0050 & 0.0094 & 0.0098 & 0.0099 & $\rightarrow$ & 0.0010 \\
\hline$(1 / 3,0.0)$ & 0.0050 & 0.0083 & 0.0098 & 0.0099 & $\rightarrow$ & 0.0010 \\
\hline$(2 / 3,0.0)$ & 0.0042 & 0.0066 & 0.0090 & 0.0099 & $\rightarrow$ & 0.0010 \\
\hline$(0.0,2 / 3)$ & 0.0050 & 0.0072 & 0.0072 & 0.0073 & $\rightarrow$ & 0.0073 \\
\hline \multicolumn{7}{|l|}{$-B$} \\
\hline$(0.0,0.0)$ & 0.0000 & 0.0000 & 0.0000 & 0.0000 & $\rightarrow$ & 0.0000 \\
\hline$(1 / 3,0.0)$ & 0.0000 & 0.0017 & 0.0032 & 0.0033 & $\rightarrow$ & 0.0033 \\
\hline$(2 / 3,0.0)$ & 0.0008 & 0.0033 & 0.0057 & 0.0066 & $\rightarrow$ & 0.0066 \\
\hline
\end{tabular}

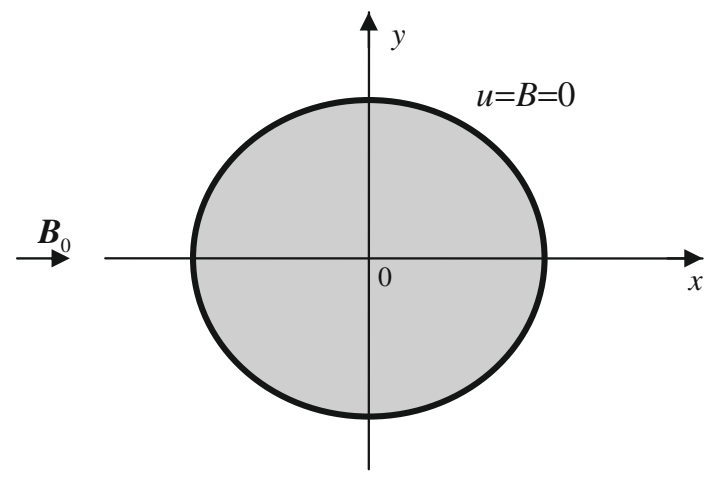

Fig. 19 Circular section of the duct with insulating walls

Table 9 CPU time and error norms for various grid size

\begin{tabular}{lccll}
\hline Hartmann & Grid & CPU time $(\mathrm{s})$ & $L_{2}$ & $L_{\infty}$ \\
\hline$M=5$ & $41 \times 41$ & 0.25 & $5.8341 \mathrm{e}-005$ & $1.2847 \mathrm{e}-004$ \\
& $81 \times 81$ & 1.4375 & $1.1781 \mathrm{e}-005$ & $3.5685 \mathrm{e}-005$ \\
& $121 \times 121$ & 4.5937 & $4.5226 \mathrm{e}-006$ & $1.6552 \mathrm{e}-005$ \\
& $161 \times 161$ & 10.7187 & $2.5827 \mathrm{e}-006$ & $1.1208 \mathrm{e}-005$ \\
$M=20$ & $41 \times 41$ & 0.1718 & $1.0355 \mathrm{e}-005$ & $2.4788 \mathrm{e}-005$ \\
& $81 \times 81$ & 1.4062 & $2.2615 \mathrm{e}-006$ & $9.6702 \mathrm{e}-006$ \\
& $121 \times 121$ & 4.6093 & $9.5792 \mathrm{e}-007$ & $5.7883 \mathrm{e}-006$ \\
& $161 \times 161$ & 10.2343 & $7.0876 \mathrm{e}-007$ & $4.6477 \mathrm{e}-006$ \\
$M=500$ & $41 \times 41$ & $0.1875 \mathrm{~s}$ & $5.5115 \mathrm{e}-005$ & $1.2056 \mathrm{e}-004$ \\
& $81 \times 81$ & 1.3750 & $2.0318 \mathrm{e}-007$ & $7.4418 \mathrm{e}-007$ \\
& $121 \times 121$ & 4.5312 & $5.3492 \mathrm{e}-009$ & $4.1434 \mathrm{e}-008$ \\
& $161 \times 161$ & 10.5625 & $5.2371 \mathrm{e}-009$ & $4.1264 \mathrm{e}-008$ \\
\hline
\end{tabular}

field was imposed at characteristic directions relative to the flow one. The LMPC method is a truly meshless method and it does not need the estimation of any integrand, while the main advantage is its simplicity. The main problem of these strong-form MFree techniques is some global or local stabil-
Table 10 CPU time and error norms for various time steps at $t=1 \mathrm{~s}$

\begin{tabular}{lllll}
\hline Hartmann & Grid & Time step & $L_{2}$ & $L_{\infty}$ \\
\hline$M=5$ & $41 \times 41$ & 0.1 & $7.7019 \mathrm{e}-005$ & $1.8504 \mathrm{e}-004$ \\
& $41 \times 41$ & 0.05 & $6.0817 \mathrm{e}-005$ & $1.3149 \mathrm{e}-004$ \\
& $41 \times 41$ & 0.025 & $6.0741 \mathrm{e}-005$ & $1.3200 \mathrm{e}-004$ \\
& $41 \times 41$ & 0.005 & $3.9113 \mathrm{e}-005$ & $8.9498 \mathrm{e}-005$ \\
$M=20$ & $41 \times 41$ & 0.1 & $5.9645 \mathrm{e}-004$ & $1.2796 \mathrm{e}-003$ \\
& $41 \times 41$ & 0.05 & $1.3864 \mathrm{e}-005$ & $3.3376 \mathrm{e}-005$ \\
& $41 \times 41$ & 0.025 & $1.1937 \mathrm{e}-005$ & $2.7987 \mathrm{e}-005$ \\
& $41 \times 41$ & 0.005 & $2.8099 \mathrm{e}-006$ & $9.1293 \mathrm{e}-006$ \\
\hline
\end{tabular}

ity issues at boundaries sites or internal points of increased complexity. These obstacles were overcame with the use of suitable nodal distributions (Type I grids), the local refinement procedure, the ensuring of positivity conditions, specifically for the case of irregular nodal distributions and the use of an interpolatory formulation for MLS approximants, which allows the direct introduction of boundary conditions, reducing the processing time and improving the condition numbers.

The numerical results of the strong-form LMPC method were compared with those obtained using two weak-form meshless methods, that is, the LBIE meshless method and the MLPG method, and with the analytical solutions, where they are available. The computed results confirm the accuracy and correctness of the proposed formulation. Velocity and induced magnetic field across the section have been evaluated at various time intervals for high Hartmann numbers (up to $10^{5}$ ) and different values of wall conductivity.

Finally, the accuracy of the method was assessed in terms of the error norms $L_{2}, L_{\infty}$, the number of nodes in the domain of influence and the time step length depicting the convergence rate of the method. Run time results were also presented demonstrating the efficiency and the applicability of the method for real world problems. 

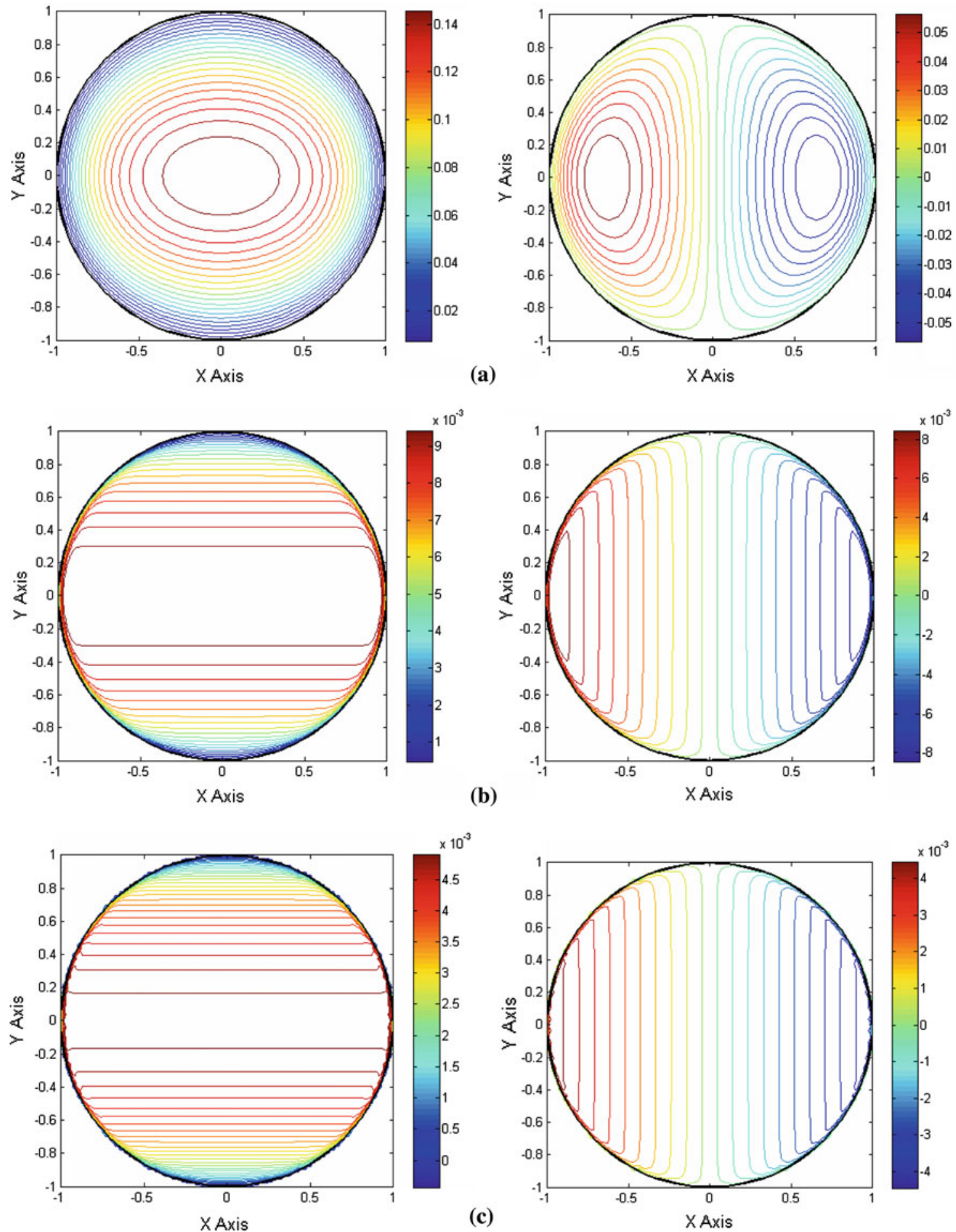

Fig. 20 Contour plots of the velocity field and the induced magnetic field for $\mathbf{a} M=5$, b $M=100$ and $\mathbf{c} M=200$ 


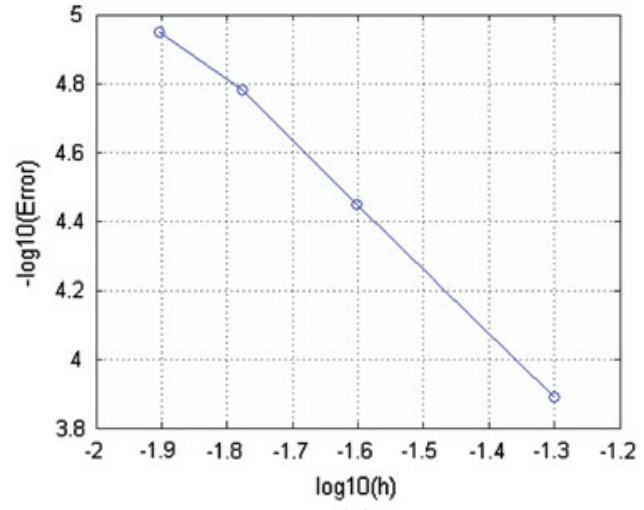

(a)

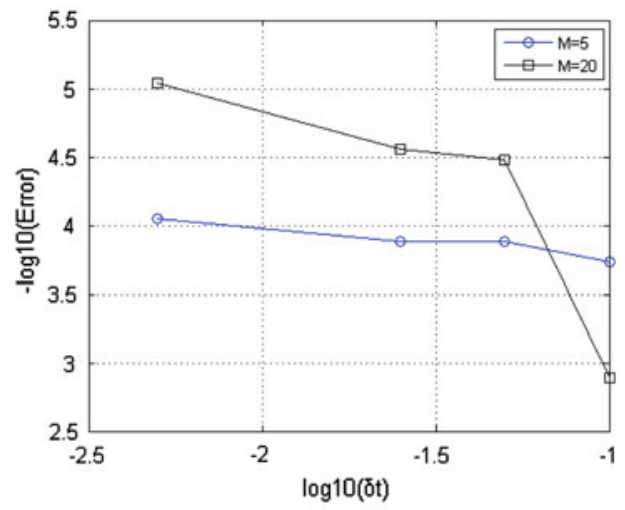

(b)

Fig. 21 Convergence rate with $\mathbf{a}$ different space steps and $\mathbf{b}$ different time steps

\section{References}

1. Hartmann J (1937) Theory of the laminar flow of an electrically conductive liquid in a homogeneous magnetic field. Math Fys Med $6: 15$

2. Gold RR (1953) Magnetohydrodynamic pipe flow Part 1. J Fluid Mech 21:577-590

3. Shercliff JA (1953) Steady motion of conducting fluids in pipes under transverse magnetic fields. Proc Camb Phil Soc 49:136-144

4. Singh B, Lal J (1978) MHD axial flow in a triangular pipe under transverse magnetic field. Ind J Pure Appl Math 9:101-115

5. Singh B, Lal J (1979) MHD axial flow in a triangular pipe under transverse magnetic field parallel to a side of the triangle. Ind $\mathrm{J}$ Technol 17:184-189

6. Singh B, Lal J (1982) Finite element method in MHD channel flow problems. Int J Numer Methods Eng 18:1104-1111

7. Singh B, Lal J (1984) Finite element method of MHD channel flow with arbitrary wall conductivity. J Math Phys Sci 18:501-516

8. Gardner LRT, Gardner GA (1995) A two-dimensional bi-cubic B-spline finite element used in a study of MHD duct flow. Comput Methods Appl Mech Eng 124:365-375

9. Tezer-Sezgin M, Köksal S (1989) Finite element method for solving MHD flow in a rectangular duct. Int J Numer Methods Eng 28:445-459

10. Demendy Z, Nagy T (1997) A new algorithm for solution of equations of MHD channel flows at moderate Hartmann numbers. Acta Mech 123:135-149

11. Barrett KE (2001) Duct flow with a transverse magnetic field at high Hartmann numbers. Int J Numer Methods Eng 50:18931906

12. Bozkaya C, Tezer-Sezgin M (2007) Fundamental solution for coupled magnetohydrodynamic flow equations. J Comput Appl Math 203:125-144

13. Singh B, Agarwal PK (1984) Numerical solution of a singular integral equation appearing in MHD. ZAMP 35:760-769

14. Tezer-Sezgin M (1994) BEM solution of MHD flow in a rectangular duct. Int J Numer Methods Fluids 18:937-952

15. Liu HW, Zhu SP (2002) The dual reciprocity boundary element method for magnetohydrodynamic channel flows. ANZIAM J 44:305-322

16. Tezer-Sezgin M, Aydin SH (2002) Dual reciprocity BEM for MHD flow using radial basis functions. Int J Comput Fluid Dyn 16:49-63

17. Carabineanu A, Dinu A, Oprea I (1995) The application of the boundary element method to the magnetohydrodynamic duct flow. ZAMP 46:971-981
18. Nesliturk AI, Tezer-Sezgin M (2005) The finite element method for MHD flow at high Hartmann numbers. Comput Methods Appl Mech Eng 194:1201-1224

19. Singh B, Lal J (1984) Finite element method for unsteady MHD flow through pipes with arbitrary wall conductivity. Int J Numer Methods Fluids 4:291-302

20. Salah NB, Soulaimani A, Habashi WG (2001) A finite element method for magnetohydrodynamic. Comput Methods Appl Mech Eng 190:5867-5892

21. Seungsoo L, Dulikravich GS (1991) Magnetohydrodynamic steady flow computation in three dimensions. Int $\mathrm{J}$ Numer Methods Fluids 13:917-936

22. Sheu TWH, Lin RK (2004) Development of a convectiondiffusion-reaction magnetohydrodynamic solver on non-staggered grids. Int J Numer Methods Fluids 45:1209-1233

23. Bozkaya C, Tezer-Sezgin M (2006) Boundary element method solution of unsteady magnetohydrodynamic duct flow with differential quadrature time integration scheme. Int J Numer Methods Fluids 51:567-584

24. Belytschko T, Krongauz Y, Organ D, Fleming M, Krysl P (1996) Meshless methods; an overview and recent developments. Comput Meth Appl Mech Eng 139:3-47

25. Bourantas GC, Skouras ED, Loukopoulos VC, Nikiforidis GC (2009) An accurate, stable and efficient domain-type meshless method for the solution of MHD flow problems. J Comput Phys 228:8135-8160

26. Verardi SLL, Machado JM, Shiyou Y (2003) The application of interpolating MLS approximations to the analysis of MHD flows. Fin Elem Anal Design 39:1173-1187

27. Dehghan M, Mirzaei D (2009) Meshless local boundary integral equation (LBIE) method for the unsteady magnetohydrodynamic (MHD) flow in rectangular and circular pipes. Comput Phys Commun 180:1458-1466

28. Dehghan M, Mirzaei D (2009) Meshless local Petrov-Galerkin (MLPG) method for the unsteady magnetohydrodynamic (MHD) flow through pipe with arbitrary wall conductivity. Appl Numer Math 59:1043-1058

29. Liu GR, Gu YT (2005) An introduction to Meshfree methods and their programming. Springer, Berlin

30. Aluru NR (2000) A point collocation method based on reproducing kernel approximations. Int J Numer Methods Eng 47:1083-1121

31. Dragos L (1975) Magneto-fluid dynamics. Academic Press, England

32. Liu GR (2002) Mesh free methods, moving beyond the finite elements method. CRC Press, Boca Raton 
33. Lancaster P, Salkauskas K (1981) Surface generated by moving least squares methods. Math Comp 37:141-158

34. Wang JG, Liu GR (2002) A point interpolation meshless method based on radial basis functions. Int J Numer Methods Eng 54: $1623-1648$

35. Kim YS, Kim DW, Jun SK, Lee JH (2007) Meshfree point collocation method for the stream-vorticity formulation of 2D incompressible Navier-Stokes equations. Comput Methods Appl Mech Eng 196:3095-3109

36. Kim DW, Kim YS (2003) Point collocation methods using the fast moving least square reproducing kernel approximation. Int $\mathbf{J}$ Numer Methods Eng 56:1445-1464

37. Bourantas GC, Skouras ED, Nikiforidis GC (2009) Adaptive support domain implementation on the moving least squares approximation for mfree methods applied on elliptic and parabolic pde problems using strong-form description. Comput Model Eng Sci 43:1-25

38. Kim DW, Liu WK (2006) Maximum principle and convergence analysis for the meshfree point collocation method. Siam J Numer Anal 44:515-539

39. Gu YT, Liu GR (2006) Meshless techniques for convection dominated problems. Comput Mech 38:171-182

40. Tezer-Sezgin M, Aydın SH (2006) Solution of magnetohydrodymanic flow problems using the boundary element method. Eng Anal Bound Elem 30:411-418 